\title{
The Economic Impact of New Firms in Punjab
}

\section{Azam Chaudhry* and Maryiam Haroon**}

\begin{abstract}
Despite the consensus that new firms have a significant economic and socioeconomic impact, there is very little empirical evidence to support this claim in the Pakistani context. In this paper, we start by looking at how new firm entry varies across districts in Punjab over time. We then look at how the establishment of different types of firms across these districts has affected district-level socioeconomic outcomes in the province. We find that firm entry has a positive impact on economic outcomes such as employment and enrollment, and that this impact can vary by the scale of the firms that enter.
\end{abstract}

Keywords: Firms, entry, Punjab, Pakistan.

JEL classification: M13, O47.

\section{Introduction}

Discussions on economic growth and socioeconomic development are closely interlinked. One aspect of this debate that appears in much of the theoretical and empirical literature is the idea that new firms create employment opportunities and growth, followed by socioeconomic development. There is, however, little empirical evidence on the actual socioeconomic impact of new firms on economic growth, except for macroeconomic analyses that look at how the overall level of industrial activity affects overall growth rates.

There are many problems with this approach. First, when one looks at country-level industrial activity and economic growth, one ignores the differences across regions. It is very possible that industrial activity has a significantly different impact in one region compared to another, both because of the characteristics of the region and the characteristics of the industries located there. Second, it is very difficult to determine if industrial activity causes development, is caused by development, or (as is most likely) if both cause each other. Higher industrial output in a region

\footnotetext{
* Professor and Dean, Department of Economics, Lahore School of Economics, Pakistan.

** Teaching Fellow and PhD student, Department of Economics, Lahore School of Economics.
} 
can cause income levels to rise, which in turn increases enrollment rates, providing more skilled labor for industries and consequently attracting new industries to enter the region. Finally, macroeconomic analyses tend to focus on overall industrial output as opposed to the nature of the firms producing this output. So, economists have looked at the relationship between the value of industrial output and economic growth, but ignored the types of firms that produce this output.

In this paper, we use a unique dataset for Punjab to examine how the entry of different types of firms in a district over time affects socioeconomic outcomes. In particular, we see how firm entry affects district-level economic outcomes such as industrial employment, primary school enrollment rates, and the number of new hospitals. We take the analysis a step further by disentangling the impact of different types of firms (small, medium, and large) on these economic outcomes. We also look at how the entry of firms that produce export goods affects these outcomes compared to those producing goods for the local market. The premise here is that the entry of different types of firms has differing impacts on development.

Although we employ a standard empirical approach as used in the literature, one has to be cautious at the outset in drawing conclusions about economic causality. So, if we find that the entry of large industrial firms has an impact on primary enrollment rates across districts in Punjab, we cannot say with absolute certainty that this is the only factor that has caused primary enrollment to rise. There could be a host of other endogenous and exogenous factors affecting both simultaneously, even if we prove that primary enrollment rates are positively correlated with the entry of large firms across districts. That said, our analysis adds to the discussion on the socioeconomic impact of industrial activity in the Pakistani context.

The setup of this paper is as follows. Section 2 looks briefly at the geographic distribution of employment and firms by size in Punjab. Section 3 reviews the literature on measuring the impact of new firm entry on employment. Section 4 presents the methodology followed. Section 5 gives the results of the empirical analysis and Section 6 discusses these results.

\section{Geographic Distribution of Industrial Employment and Firms by Size in Punjab}

We begin by looking at maps that explain the regional breakdown of industrial employment and firm distribution by size across districts of 
Punjab. For these maps, we use the Government of Punjab's Directory of Industries (DOI) for 2010, 2006, and 2002; this is a firm-level dataset that covers more than 18,000 manufacturing firms.

The district-level breakdown of industrial employment is given in Figure 1 as a ratio of total employment in the province for 2010, 2006, and 2004. The darker-shaded districts have a higher share of industrial employment. In 2010, the greatest share of industrial employment was concentrated in districts such as Lahore, Kasur, Faisalabad, Sheikhupura, Gujranwala, Sialkot, and Gujrat; districts such as Pakpattan, Layyah, Lodhran, Bhakkar, and Mianwali had a smaller share of industrial employment. This distribution of employment in the industrial sector was approximately the same in 2004 and 2006.

Figure 2 indicates which districts had the highest share of small, medium, and large firms as a proportion of total firms in the district. In particular, we see that, in 1995-2010, the highest concentration of small firms was in Sialkot, Hafizabad, Gujranwala, Toba Tek Singh, Okara, and Pakpattan. The largest concentration of medium firms was in Rawalpindi and in certain districts in southern Punjab (Dera Ghazi Khan, Rajanpur, and Rahimyar Khan). Finally, the highest concentration of large firms was primarily in central Punjab in Lahore, Faisalabad, and Sheikhupura.

Figure 3 shows the breakdown of districts with the highest proportion of firms producing goods for export. This breakdown reinforces what is generally known: that most export good producers are in central Punjab in districts such as Lahore, Faisalabad, Sialkot, Gujranwala, and Kasur. Comparing these maps, we also see that those districts with the highest proportion of exporting firms account for the highest employment shares. 
Figure 1: District-level breakdown of industrial employment, Punjab
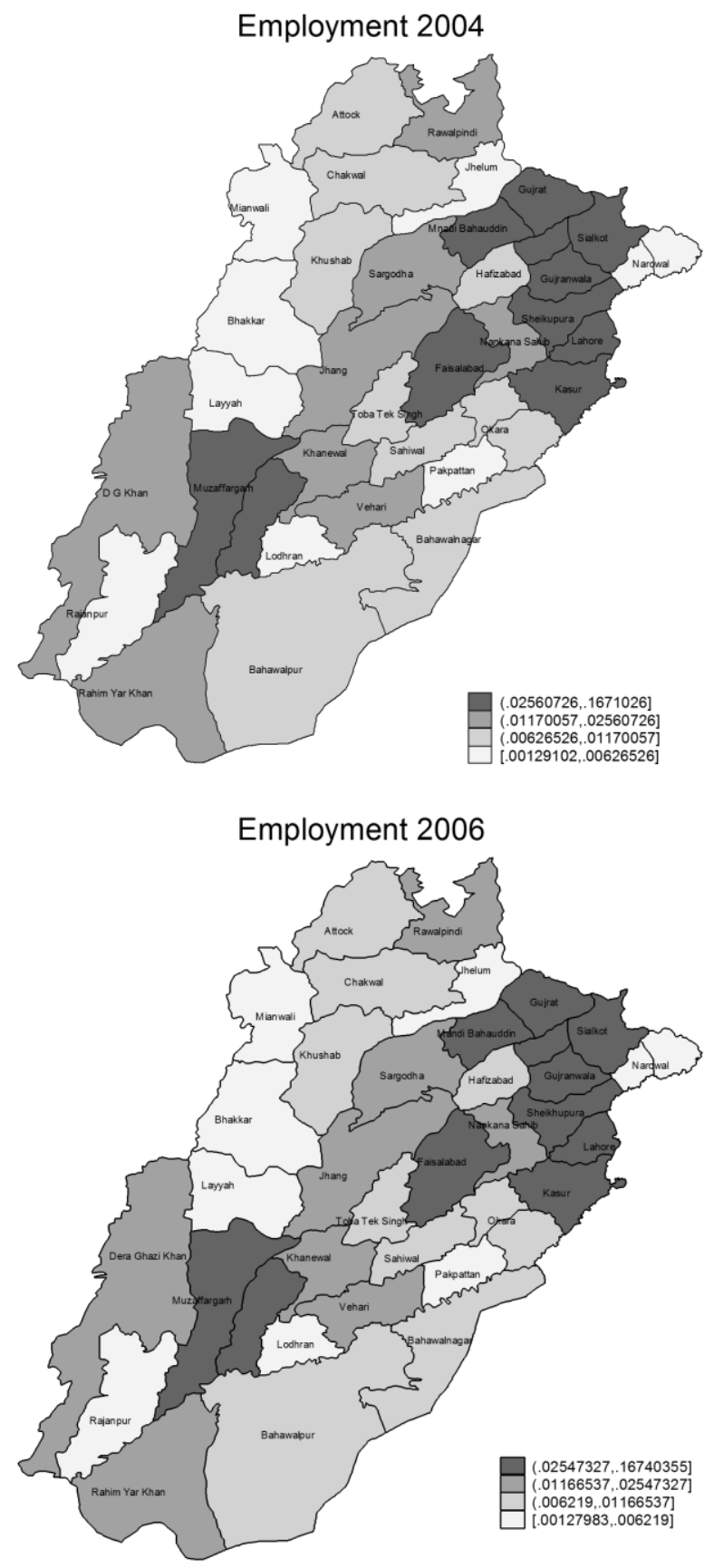


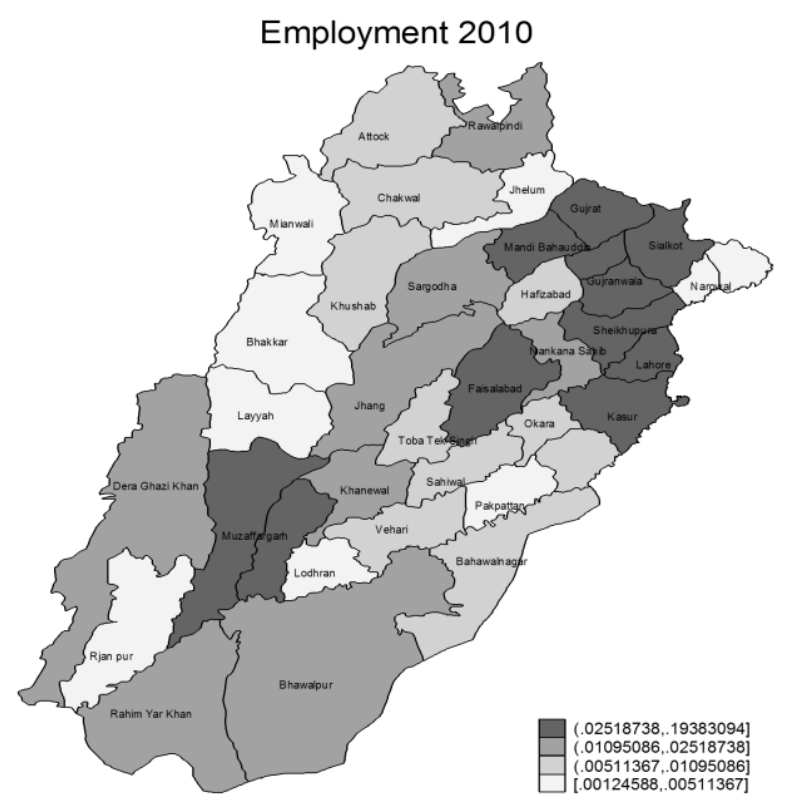

Figure 2: Districts with the highest share of small, medium, and large firms as a proportion of total firms, Punjab

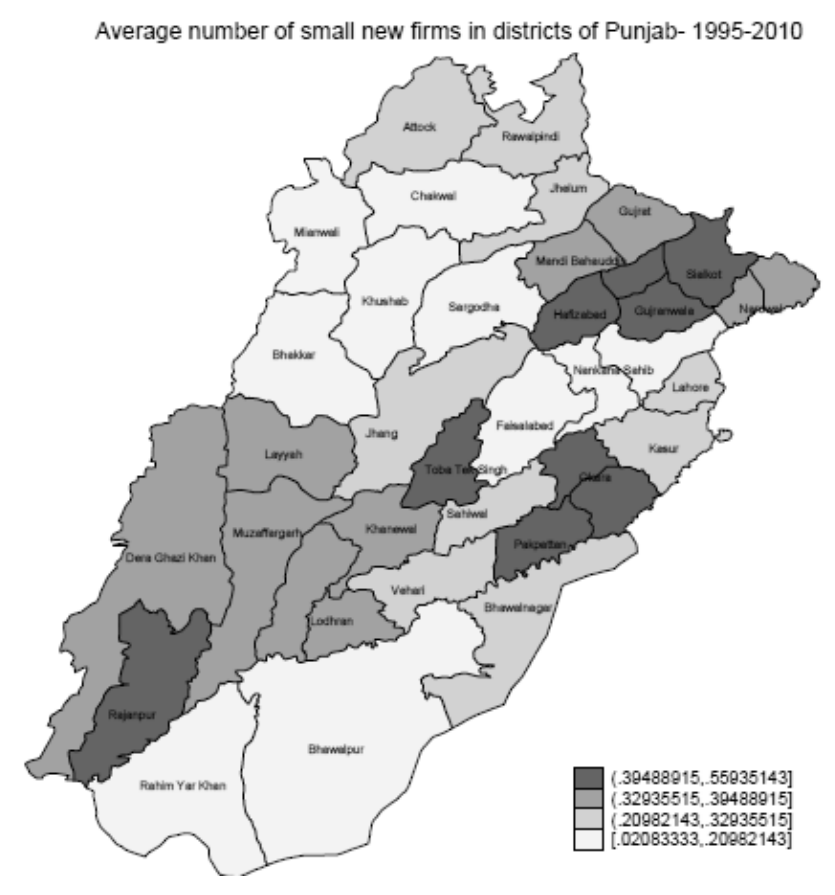


Average number of medium new firms in districts of Punjab -1995-2010

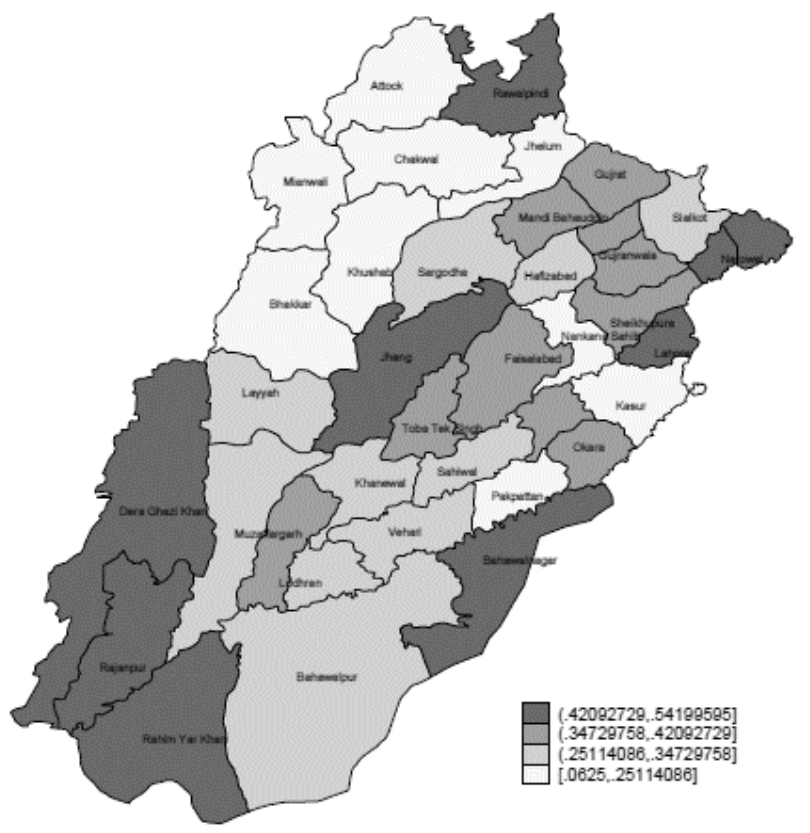

Average number of large new firms in districts of Punjab - 1995-2010

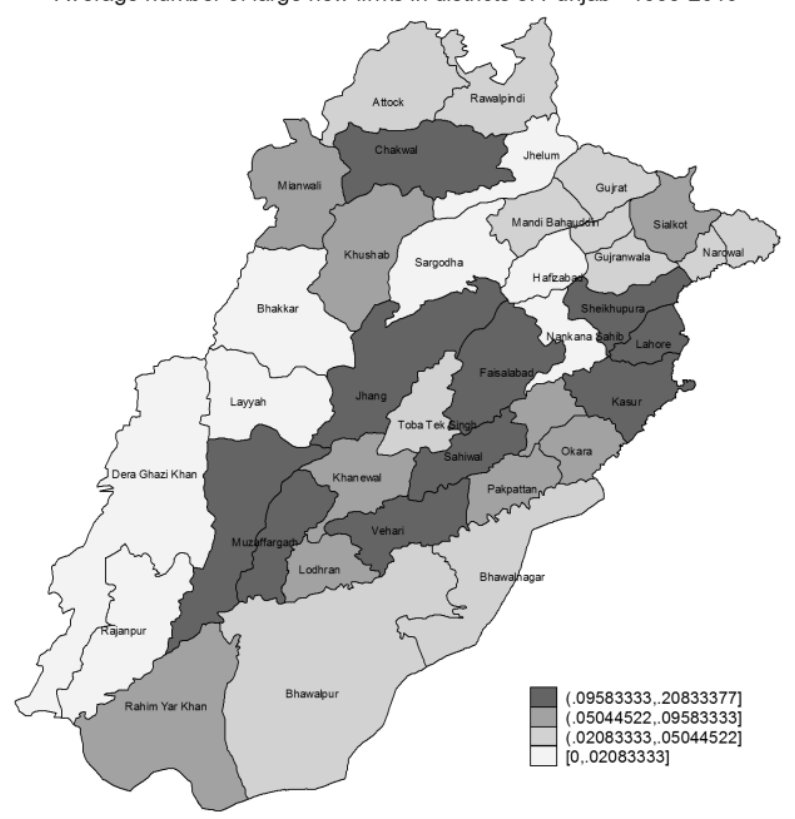


Figure 3: Districts with the highest proportion of export goodproducing firms, Punjab

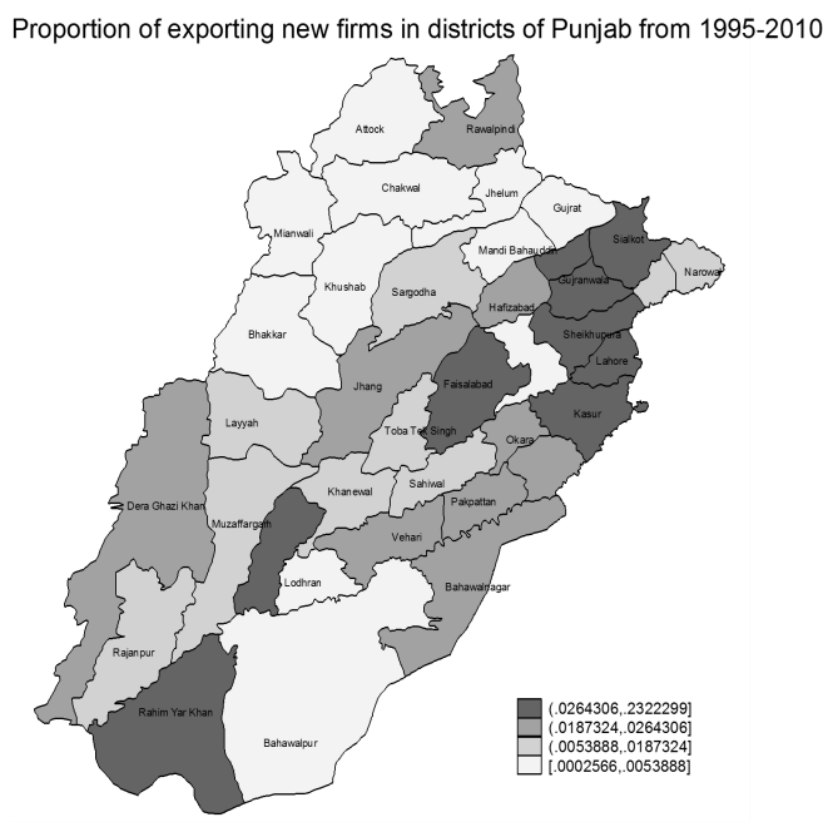

\section{Literature Review}

The impact of new firm entry on regional development is complex because there are numerous factors at play. Apart from the direct effects of firms entering a market, such as higher output and employment, there are myriad indirect effects, such as larger or more competitive markets after firm entry (or the opposite if a new firm enters and eliminates competition), more innovation as a result of new firm entry, greater variety and quality of products, and the development of ancillary goods and services. Moreover, it is extremely difficult to separate out the impact of macroeconomic factors on regional development from the impact of firm entry: if regional employment goes up, is it because of some positive macroeconomic shock that may affect regions differently or is it because of new firm entry? The most likely answer is that it is a combination of both, with each of these factors affecting the other; this makes the process of isolating the impact of firm entry on regional development difficult.

For this reason, the literature on new firm entry is varied: some studies look at the impact of economic fluctuations on regional growth (see Callejón \& Segarra, 2000; Bosma \& Nieuwenhuijsen, 2002; Caves, 1998), while others examine the impact of firm entry and economic fluctuations 
on different economic sectors such as manufacturing and services (see Acs \& Armington, 2003; Bosma \& Nieuwenhuijsen, 2002; Geroski, 1995). The more recent literature focuses on the impact of firm entry across regions on regional employment (or unemployment).

Acs and Armington (2003) and Reynolds (1994, 1999) look at the impact of firm entry on regional employment changes in the US. They find the impact is significant, though varied over time. Similarly, Ashcroft and Love (1996) and Mueller, van Stel, and Storey (2008) find that the impact of firm entry on regional employment in the UK varies by region. Mueller et al. (2008) conclude that this impact is positive and significant for England, but not significant for Scotland. Foelster (2000) finds that firm entry has a significantly positive impact on self-employment rates in Sweden. Brixy (1999) shows that new firm entry had a significant impact on regional employment in East Germany early on after reunification, while van Stel and Suddle (2008) find that new firm entry has a significant impact on changes in regional employment in the Netherlands.

What differentiates the literature on regional economic growth and new firm entry from that on regional employment changes and new firm entry is that the latter focuses on how the impact of new firm entry can change over time: new firms entering today may have a different impact compared to one, two, or three years from now. Fritsch and Mueller (2004, 2007), who were among the first to look at the lagged effects of firm entry on regional employment, explain that, when a firm enters a market, it can have different impacts on regional development at different points in time. The "direct" positive effect on employment may be followed by a "displacement" effect whereby new firm entry can lead to the exit of other firms (due to differences in productivity, scale, and technology), in turn causing employment to fall. Finally, the firm's entry can potentially stimulate surviving firms into performing better and expanding; this "induced" effect increases employment.

Fritsch and Mueller $(2004,2007)$ look at the differential impact over time of firm entry by testing if regional employment is a function of the present and lagged values of firm entry. By regressing changes in regional employment rates on these values, they determine how the impact of any firm entering a market is different today compared to one year from now, two years from now, and so on. More recent work has started looking at how the entry of different types of firms affects employment. Baptista and Preto (2011) show that the impact on employment of knowledge-based firm entry is different from that of other firms, while van Stel and Suddle 
(2008) look at the differential impact on employment of manufacturing visà-vis nonmanufacturing firms entering regional markets.

Our work takes this literature and extends it, making the study one of the first attempts to look at the impact of firm entry on changes in employment rates in a developing country context. In order to control for regional heterogeneity, we include regional fixed effects. We also look at the impact on regional employment of overall firm entry as well as breaking these firms down into small, medium, and large firms and into exporting versus nonexporting firms. In other words, we see if small firms entering a regional market have a different impact on regional employment compared to medium and large firms, and whether the entry of exporting firms has a different impact on regional employment compared to nonexporting firms.

We take a different route from the rest of the literature. Arguing that new firm entry can have a significant impact not just on regional employment, but also on regional socioeconomic development in a country such as Pakistan, we look at the impact of new firm entry on other regional characteristics, including primary school enrollment, the number of primary schools, and the number of hospitals. We do this to see if new firm entry has spillover effects from employment to household outcomes.

This may be considered a more tenuous series of relationships than just the impact of firm entry on employment (which is relatively straightforward) because so many more unobserved (at least in this case) factors may affect some of these socioeconomic variables. However, even if we cannot prove causality, we can at least prove correlation: we might not be able to say definitively that new firm entry causes a rise or fall in primary school enrollment at the regional level, but at least we can say that new firm entry is correlated with a rise or fall in primary school enrollment. This in itself is important from both the academic's point of view as well as from the policymaker's point of view.

\section{Methodology}

The DOI dataset for 2010, 2006, and 2002 includes information on each firm's year of establishment, employment level, initial investment, location, product manufactured, and industry. We use these data to construct measures of the district-level growth in employment, firm birth, and average firm size. We also use the Punjab Development Statistics dataset for 2006 to 2012 (collected by the Punjab Bureau of Statistics) on health indicators, education indicators, population, and the area of each 
district. The employment data it provides is used as a robustness check for estimations.

The empirical analysis follows the standard estimation procedure discussed in the firm entry and regional development literature. The dependent variables are growth in employment or primary enrollment. The independent variables are the contemporaneous rate of firm entry and lagged values of firm entry. The control variables include population density and average firm size in a district.

The first set of regressions includes the standard regressions that measure the impact of firm entry over time on district-level industrial employment. For this reason, we take the growth rate in industrial employment in the districts of Punjab over time as the dependent variable and the number of firms that have entered each district in this period. As discussed above, the standard methodology is to use lagged values of the number of firms that have entered each district over time as well as a fixed effect to control for district-level fixed effects. As per standard practice, we use up to 10 lags of the independent variable to take into account the possibility that a firm entering in one year can affect employment in the following years.

The second set of regressions deviates from the literature in an interesting way. Since we know that small firm entry has a different impact on employment compared to large firm entry - not only because of the obvious difference in the number of workers employed by different sized firms, but because of the spillover effects from firm entry, such as increased demand for ancillary goods and services - we divide district-level firm entry over the time period into the entry of small, medium, and large firms (characterized by employment). This allows us to separate out the effects of firm entry based on firm size. In this set of regressions, we also control for district-level differences by including fixed effects as well as lagged values of the number of firms entering each district.

The third set of regressions looks at the differing impact of the entry of firms that produce goods for export compared to firms that produce goods for the domestic market. As Chaudhry and Haseeb (2014) show, exporting firms tend to be different from nonexporting firms in terms of productivity and size, and so, we estimate the effect of district-level firm entry on the growth in employment. As above, we include lagged values of the number of firms that have entered as well as fixed effects. 
The fourth set of regressions deviates liberally from the standard literature: in the context of Pakistan, we argue that firm entry not only impacts employment, but also other socioeconomic outcomes such as education and health. For example, there is a significant body of literature showing how the creation of firms can increase school enrollment through higher incomes and other factors, such as parents wanting to educate their children so they are able to find manufacturing jobs. At the same time, many studies indicate that the entry of new firms can lead to higher levels of child labor, which in turn can decrease enrollment rates. To test for this, we estimate the impact of firm entry at the district level on district-level primary school enrollment and number of schools. Again, we include fixed effects and lagged values of the independent variable.

Similarly, the fifth set of regressions looks at how healthcare is affected by firm entry. As in the regressions above, we test to see if the growth in number of hospitals is a function of the entry of new firms in a district over time. We extend these basic models by testing if primary school enrollment and the number of primary schools are differentially affected by the size of the firm entering (small, medium, or large) in our sixth model, and by testing if the district-level number of hospitals is differentially affected by the size of the firm entering (small, medium, or large) in our seventh model.

Finally, the last set of regressions determines the impact on school enrollment and the number of schools over time of the entry of firms producing exportable goods across districts in Punjab.

\section{Results}

The first set of regressions focuses on the overall impact of firm entry on employment growth across districts in Punjab (Table 1). As the results show, the average size of entrants has a negative relationship with growth in employment, which means that, as larger firms enter a district, employment growth decreases. The results also show that, at an aggregate level, firm entry does not affect employment over the time period. 
Table 1: Impact of firm entry on employment across districts in Punjab

\begin{tabular}{|c|c|}
\hline Variable & Employment growth \\
\hline Population density & $\begin{array}{l}0.00696 \\
(0.0157)\end{array}$ \\
\hline Average firm size & $\begin{array}{l}-0.105^{* *} \\
(0.0509)\end{array}$ \\
\hline Firm birth $\mathrm{t}$ & $\begin{array}{c}1.437 \\
(3.488)\end{array}$ \\
\hline Firm birth $\mathrm{t}-1$ & $\begin{array}{c}3.096 \\
(4.119)\end{array}$ \\
\hline Firm birth $\mathrm{t}-2$ & $\begin{array}{c}3.935 \\
(4.970)\end{array}$ \\
\hline Firm birth $\mathrm{t}-3$ & $\begin{array}{l}-2.802 \\
(3.978)\end{array}$ \\
\hline Firm birth $\mathrm{t}-4$ & $\begin{array}{l}-0.481 \\
(3.652)\end{array}$ \\
\hline Firm birth $\mathrm{t}-5$ & $\begin{array}{c}2.057 \\
(3.515)\end{array}$ \\
\hline Firm birth $\mathrm{t}-6$ & $\begin{array}{l}-2.168 \\
(3.588)\end{array}$ \\
\hline Firm birth $\mathrm{t}-7$ & $\begin{array}{l}-2.277 \\
(4.196)\end{array}$ \\
\hline Firm birth $\mathrm{t}-8$ & $\begin{array}{l}-0.266 \\
(4.476)\end{array}$ \\
\hline Firm birth $\mathrm{t}-9$ & $\begin{array}{c}0.450 \\
(4.223)\end{array}$ \\
\hline Firm birth $\mathrm{t}-10$ & $\begin{array}{l}1.603 \\
(5.835)\end{array}$ \\
\hline Constant & $\begin{array}{c}3.513 \\
(11.47)\end{array}$ \\
\hline R-squared & 0.064 \\
\hline District fixed effects & Yes \\
\hline
\end{tabular}

Note: Standard errors in parentheses.

${ }^{* * *} \mathrm{p}<0.01,{ }^{* *} \mathrm{p}<0.05,{ }^{*} \mathrm{p}<0.1$

Dependent variable

Employment growth $=$ (employment in region $i$ at time $t-$ employment in region $i$ at time $t-2$ )/employment in region $i$ at time $t-2$

Independent variables

Firm birth $=($ new firms in region $i$ at time $t /$ total new firms in Punjab at time $t)$

Population density $=$ (population in region $i$ at time $t /$ area of a region)

Average firm size $=($ average size of firms in region $i$ at time $t)$

Source: Authors' calculations. 
Figure 4 illustrates the impact of overall firm entry on districtlevel employment in Punjab. We see a slight rise in district-level employment after firm entry, followed by a fall and then a rise. This may reflect the idea discussed above concerning the fluctuating impact of firm entry on employment over time. As mentioned, the impact is not statistically significant.

Figure 4: Impact of all firm entry on district-level employment in Punjab

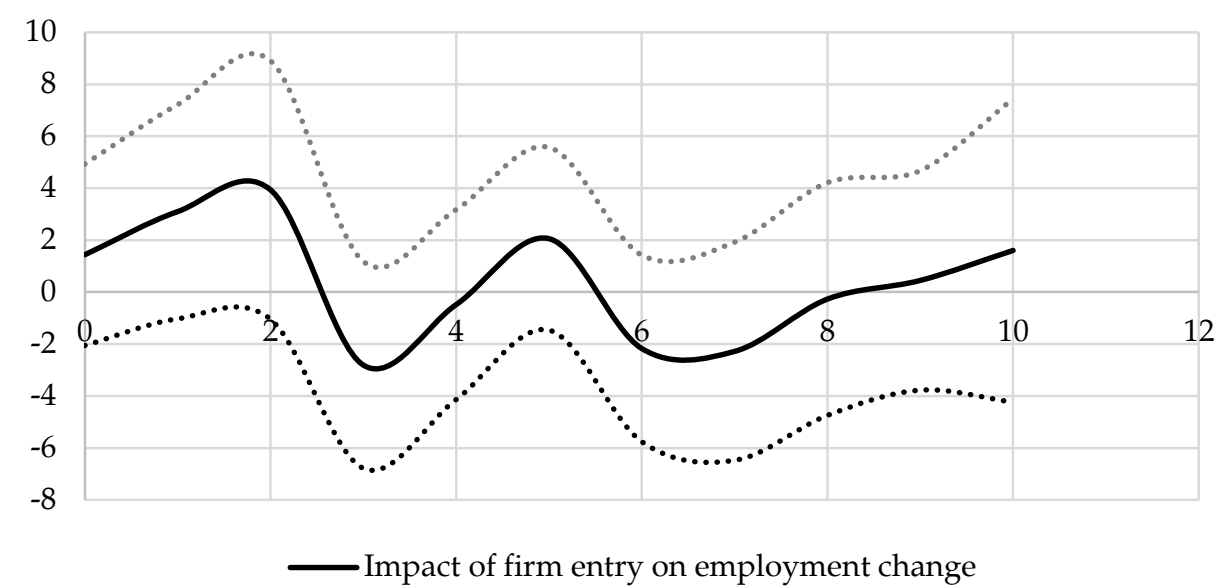

Note: The x-axis shows the number of time lags of firm entry or, in other words, the impact of firm entry one year later, two years later, etc. The y-axis shows the percentage change.

Source: These figures are generated from the regression results shown above, based on data from the Punjab Directory of Industries.

The second set of results shows the impact of firm entry on employment growth when firms are broken down into small, medium, and large enterprises. Columns 1 to 3 in Table 2 show the impact of small, medium, and large firm entry on employment growth across districts. Column 4 indicates the impact of all firms simultaneously on employment growth across districts. The results show that small and medium firms are associated with higher employment growth at the district level. The combined specification shows that the entry of large firms does not lead to higher employment growth over time.

Looking more closely at the coefficients of the lagged variables, we see that, on average, it takes about three years for small firm entry to increase employment growth and a year for the entry of medium firms to do so, although the employment impact of small firms is larger. So the entry of small firms has had the greatest impact on employment growth across districts in Punjab, but this impact occurs faster when medium firms enter. 
Table 2: Impact of small, medium, and large firm entry on employment across districts in Punjab

\begin{tabular}{|c|c|c|c|c|c|c|}
\hline \multirow[b]{2}{*}{ Variable } & \multicolumn{6}{|c|}{ Employment growth } \\
\hline & (1) & (2) & (3) & (4) & (5) & (6) \\
\hline \multirow{2}{*}{ Population density } & -0.00343 & 0.00599 & 0.0102 & 0.00381 & 0.00709 & 0.0151 \\
\hline & $(0.0166)$ & $(0.0151)$ & $(0.0164)$ & $(0.0145)$ & $(0.0193)$ & $(0.0193)$ \\
\hline \multirow[t]{2}{*}{ Average firm size } & $-0.0987^{* *}$ & $-0.114^{* *}$ & $-0.120^{* *}$ & $-0.140^{* * *}$ & -0.0802 & $-0.0978^{*}$ \\
\hline & $(0.0465)$ & $(0.0461)$ & $(0.0494)$ & $(0.0483)$ & $(0.0507)$ & $(0.0527)$ \\
\hline \multirow[t]{2}{*}{ Small firm birth $\mathrm{t}$} & 3.423 & & & & $4.687^{*}$ & $4.758^{* *}$ \\
\hline & $(2.278)$ & & & & $(2.383)$ & (2.403) \\
\hline \multirow[t]{2}{*}{ Small firm birth $\mathrm{t}-1$} & 3.118 & & & & $4.471^{*}$ & $4.222^{*}$ \\
\hline & $(2.189)$ & & & & $(2.291)$ & $(2.295)$ \\
\hline \multirow[t]{2}{*}{ Small firm birth $\mathrm{t}-2$} & -3.261 & & & & -1.673 & -1.340 \\
\hline & $(2.247)$ & & & & (2.529) & $(2.550)$ \\
\hline \multirow[t]{2}{*}{ Small firm birth $\mathrm{t}-3$} & $6.118^{* * *}$ & & & & $5.349^{* *}$ & $5.339 * *$ \\
\hline & $(2.031)$ & & & & $(2.253)$ & $(2.284)$ \\
\hline \multirow[t]{2}{*}{ Small firm birth $\mathrm{t}-4$} & -1.655 & & & & -0.950 & -0.334 \\
\hline & $(1.874)$ & & & & (2.143) & (2.197) \\
\hline \multirow{2}{*}{ Small firm birth $t-5$} & 0.904 & & & & 0.643 & 1.397 \\
\hline & $(1.800)$ & & & & (2.199) & $(2.286)$ \\
\hline \multirow{2}{*}{ Small firm birth $\mathrm{t}-6$} & -0.389 & & & & -0.0267 & 0.242 \\
\hline & $(1.682)$ & & & & (1.953) & (1.989) \\
\hline \multirow[t]{2}{*}{ Small firm birth $\mathrm{t}-7$} & 0.00645 & & & & 1.195 & 1.398 \\
\hline & $(1.517)$ & & & & $(1.741)$ & $(1.750)$ \\
\hline \multirow[t]{2}{*}{ Small firm birth $\mathrm{t}-8$} & -0.0842 & & & & -0.134 & 0.836 \\
\hline & $(1.607)$ & & & & (1.917) & $(1.977)$ \\
\hline \multirow[t]{2}{*}{ Small firm birth $t-9$} & -1.917 & & & & -1.028 & -1.780 \\
\hline & $(1.627)$ & & & & (1.997) & $(2.038)$ \\
\hline \multirow[t]{2}{*}{ Small firm birth $\mathrm{t}-10$} & 1.460 & & & & -0.475 & -0.564 \\
\hline & $(1.739)$ & & & & $(2.346)$ & $(2.367)$ \\
\hline \multirow[t]{2}{*}{ Medium firm birth $t$} & & 0.570 & & & 0.830 & 1.467 \\
\hline & & $(1.400)$ & & & $(1.534)$ & (1.633) \\
\hline \multirow[t]{2}{*}{ Medium firm birth $\mathrm{t}-1$} & & $4.286^{* * *}$ & & & $3.570^{* *}$ & $3.756^{* *}$ \\
\hline & & $(1.491)$ & & & $(1.649)$ & $(1.695)$ \\
\hline \multirow[t]{2}{*}{ Medium firm birth $\mathrm{t}-2$} & & $3.571^{* *}$ & & & 1.733 & 1.963 \\
\hline & & $(1.618)$ & & & $(1.822)$ & $(1.880)$ \\
\hline \multirow[t]{2}{*}{ Medium firm birth $\mathrm{t}-3$} & & -0.371 & & & -1.880 & -1.054 \\
\hline & & (1.668) & & & (1.836) & (1.907) \\
\hline \multirow[t]{2}{*}{ Medium firm birth $\mathrm{t}-4$} & & 0.798 & & & -0.748 & 0.791 \\
\hline & & (1.773) & & & $(2.027)$ & $(2.087)$ \\
\hline \multirow[t]{2}{*}{ Medium firm birth $\mathrm{t}-5$} & & 0.472 & & & -0.667 & 0.769 \\
\hline & & $(1.716)$ & & & (2.023) & $(2.076)$ \\
\hline
\end{tabular}




\begin{tabular}{|c|c|c|c|c|c|c|}
\hline \multirow[b]{2}{*}{ Variable } & \multicolumn{6}{|c|}{ Employment growth } \\
\hline & (1) & (2) & (3) & (4) & (5) & (6) \\
\hline Medium firm birth $\mathrm{t}-6$ & & $\begin{array}{l}1.802 \\
(1.697)\end{array}$ & & & $\begin{array}{l}0.391 \\
(2.046)\end{array}$ & $\begin{array}{l}1.780 \\
(2.133)\end{array}$ \\
\hline Medium firm birth $\mathrm{t}-7$ & & $\begin{array}{l}2.400 \\
(1.776)\end{array}$ & & & $\begin{array}{l}2.830 \\
(2.101)\end{array}$ & $\begin{array}{l}3.785^{*} \\
(2.154)\end{array}$ \\
\hline Medium firm birth $\mathrm{t}-8$ & & $\begin{array}{l}-0.233 \\
(1.859)\end{array}$ & & & $\begin{array}{l}0.381 \\
(2.136)\end{array}$ & $\begin{array}{l}1.224 \\
(2.169)\end{array}$ \\
\hline Medium firm birth $t-9$ & & $\begin{array}{l}0.309 \\
(2.118)\end{array}$ & & & $\begin{array}{l}0.628 \\
(2.520)\end{array}$ & $\begin{array}{l}1.203 \\
(2.534)\end{array}$ \\
\hline Medium firm birth $\mathrm{t}-10$ & & $\begin{array}{l}-2.236 \\
(2.252)\end{array}$ & & & $\begin{array}{l}-2.573 \\
(2.881)\end{array}$ & $\begin{array}{l}-2.385 \\
(2.885)\end{array}$ \\
\hline Large firm birth $t$ & & & $\begin{array}{l}-2.484 \\
(3.196)\end{array}$ & & $\begin{array}{l}-1.981 \\
(3.322)\end{array}$ & $\begin{array}{l}-1.480 \\
(3.380)\end{array}$ \\
\hline Large firm birth $\mathrm{t}-1$ & & & $\begin{array}{l}-1.281 \\
(3.257)\end{array}$ & & $\begin{array}{l}0.918 \\
(3.436)\end{array}$ & $\begin{array}{l}-0.219 \\
(3.556)\end{array}$ \\
\hline Large firm birth $\mathrm{t}-2$ & & & $\begin{array}{l}-4.639 \\
(3.387)\end{array}$ & & $\begin{array}{l}-3.245 \\
(3.419)\end{array}$ & $\begin{array}{l}-4.839 \\
(3.427)\end{array}$ \\
\hline Large firm birth $\mathrm{t}-3$ & & & $\begin{array}{l}-3.853 \\
(3.566)\end{array}$ & & $\begin{array}{l}-4.074 \\
(3.608)\end{array}$ & $\begin{array}{l}-8.363^{* *} \\
(3.816)\end{array}$ \\
\hline Large firm birth $\mathrm{t}-4$ & & & $\begin{array}{l}-1.259 \\
(3.785)\end{array}$ & & $\begin{array}{l}-1.221 \\
(3.882)\end{array}$ & $\begin{array}{l}-0.773 \\
(3.923)\end{array}$ \\
\hline Large firm birth $\mathrm{t}-5$ & & & $\begin{array}{l}-2.085 \\
(3.594)\end{array}$ & & $\begin{array}{l}-2.136 \\
(3.775)\end{array}$ & $\begin{array}{l}-1.380 \\
(3.826)\end{array}$ \\
\hline Large firm birth $\mathrm{t}-6$ & & & $\begin{array}{l}-4.066 \\
(4.057)\end{array}$ & & $\begin{array}{l}-2.906 \\
(4.233)\end{array}$ & $\begin{array}{l}-2.590 \\
(4.334)\end{array}$ \\
\hline Large firm birth $\mathrm{t}-7$ & & & $\begin{array}{l}-5.588 \\
(4.217)\end{array}$ & & $\begin{array}{l}-1.635 \\
(4.466)\end{array}$ & $\begin{array}{l}-2.008 \\
(4.530)\end{array}$ \\
\hline Large firm birth $\mathrm{t}-8$ & & & $\begin{array}{l}0.117 \\
(3.951)\end{array}$ & & $\begin{array}{l}2.569 \\
(4.244)\end{array}$ & $\begin{array}{l}1.500 \\
(4.252)\end{array}$ \\
\hline Large firm birth $\mathrm{t}-9$ & & & $\begin{array}{l}1.785 \\
(4.145)\end{array}$ & & $\begin{array}{l}2.809 \\
(4.462)\end{array}$ & $\begin{array}{l}0.968 \\
(4.673)\end{array}$ \\
\hline Large firm birth $\mathrm{t}-10$ & & & $\begin{array}{l}-4.056 \\
(4.818)\end{array}$ & & $\begin{array}{l}-0.578 \\
(5.023)\end{array}$ & $\begin{array}{l}-2.194 \\
(5.202)\end{array}$ \\
\hline Other firm birth $\mathrm{t}$ & & & & $\begin{array}{l}14.59^{* *} \\
(5.762)\end{array}$ & & $\begin{array}{l}18.34^{* * *} \\
(6.218)\end{array}$ \\
\hline Other firm birth $\mathrm{t}-1$ & & & & $\begin{array}{l}19.15^{* *} \\
(7.932)\end{array}$ & & $\begin{array}{l}27.22^{* * *} \\
(8.448)\end{array}$ \\
\hline Other firm birth $\mathrm{t}-2$ & & & & $\begin{array}{l}2.896 \\
(7.746)\end{array}$ & & $\begin{array}{l}8.827 \\
(8.406)\end{array}$ \\
\hline Other firm birth $\mathrm{t}-3$ & & & & $\begin{array}{l}6.909 \\
(7.538)\end{array}$ & & $\begin{array}{l}6.027 \\
(8.366)\end{array}$ \\
\hline Other firm birth $\mathrm{t}-4$ & & & & 10.61 & & 9.298 \\
\hline
\end{tabular}




\begin{tabular}{|c|c|c|c|c|c|c|}
\hline \multirow[b]{2}{*}{ Variable } & \multicolumn{6}{|c|}{ Employment growth } \\
\hline & (1) & (2) & (3) & (4) & (5) & (6) \\
\hline & & & & $(7.778)$ & & $(8.531)$ \\
\hline \multirow[t]{2}{*}{ Other firm birth $\mathrm{t}-5$} & & & & 10.35 & & 10.44 \\
\hline & & & & $(7.502)$ & & $(8.347)$ \\
\hline \multirow[t]{2}{*}{ Other firm birth $t-6$} & & & & 6.430 & & 10.60 \\
\hline & & & & $(7.974)$ & & $(8.353)$ \\
\hline \multirow[t]{2}{*}{ Other firm birth $\mathrm{t}-7$} & & & & 2.355 & & 4.441 \\
\hline & & & & $(8.112)$ & & $(8.792)$ \\
\hline \multirow[t]{2}{*}{ Other firm birth $\mathrm{t}-8$} & & & & 7.526 & & 12.67 \\
\hline & & & & $(8.082)$ & & $(9.150)$ \\
\hline \multirow[t]{2}{*}{ Other firm birth $t-9$} & & & & 8.290 & & 2.899 \\
\hline & & & & $(10.21)$ & & (10.73) \\
\hline \multirow[t]{2}{*}{ Other firm birth $\mathrm{t}-10$} & & & & 0.0615 & & 11.21 \\
\hline & & & & $(11.46)$ & & (12.15) \\
\hline \multirow[t]{2}{*}{ Constant } & 11.14 & 0.896 & 5.062 & 7.106 & 1.544 & -6.769 \\
\hline & (12.13) & $(9.963)$ & $(10.43)$ & $(9.713)$ & $(14.00)$ & $(14.30)$ \\
\hline District fixed effects & Yes & Yes & Yes & Yes & Yes & Yes \\
\hline
\end{tabular}

Note: Standard errors in parentheses.

*** $\mathrm{p}<0.01,{ }^{* *} \mathrm{p}<0.05,{ }^{*} \mathrm{p}<0.1$

Dependent variable

Employment growth $=($ employment in region $i$ at time $t-$ employment in region $i$ at time $t-2$ )/employment in region $i$ at time $t-2$

Independent variables

Small firm birth $=($ new firms with fewer than 10 employees in region $i$ at time $t /$ total new small firms in Punjab at time $t)$

Medium firm birth $=($ new firms with 10 or more employees and fewer than 50 in region $i$ at time $t$ /total new medium firms in Punjab at time $t$ )

Large firm birth $=$ (new firms with 50 or more employees in region $i$ at time $t /$ total new large firms in Punjab at time $t$ )

Other firm birth $=($ new firms with no employment reported in region $i$ at time $t /$ total new firms whose employment is not reported in Punjab at time $t$ )

Population density $=$ (population in region $i$ at time $t$ /area of a region)

Average firm size $=($ average size of firms in region $i$ at time $t)$

Source: Authors' calculations.

These results are illustrated in Figures 5, 6, and 7. Figure 5 shows that the change in district-level employment increases as soon as small firms enter the market; this decreases and then increases once again. On average, there is a significant fall in the growth rate of employment a few years after a large firm enters the market, and this impact is greater than the employment impact of a small entrant. Finally, the entry of a medium firm tends to increase the growth rate of employment about a year after entry, though this impact dissipates after a year. 


\section{Figure 5: Impact of small firm entry on district-level employment in} Punjab

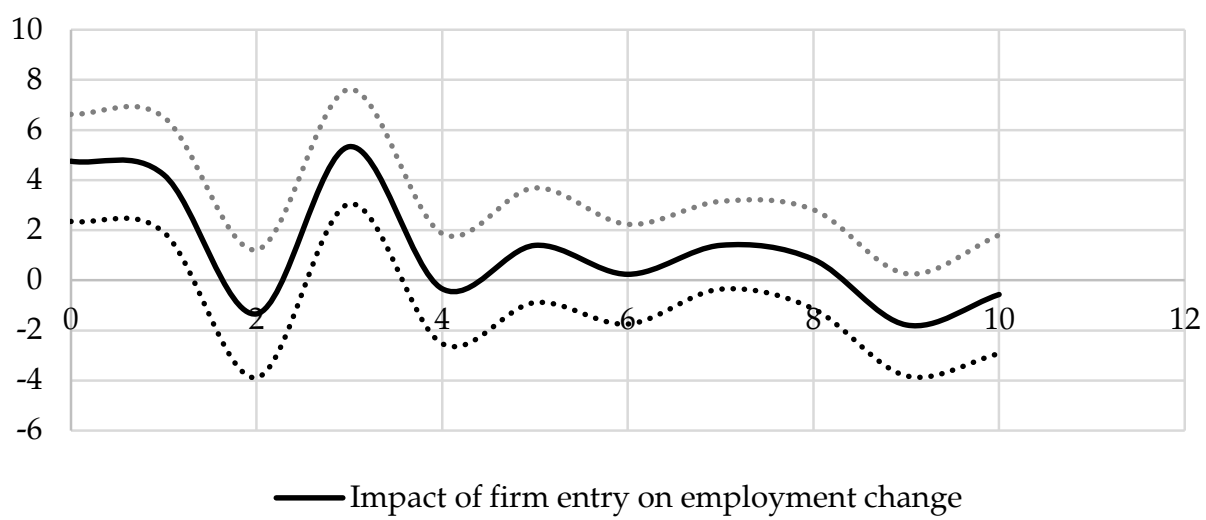

Note: The x-axis shows the number of time lags of firm entry or, in other words, the impact of firm entry one year later, two years later, etc. The y-axis shows the percentage change. Source: These figures are generated from the regression results shown above, based on data from the Punjab Directory of Industries.

Figure 6: Impact of medium firm entry on district-level employment in Punjab

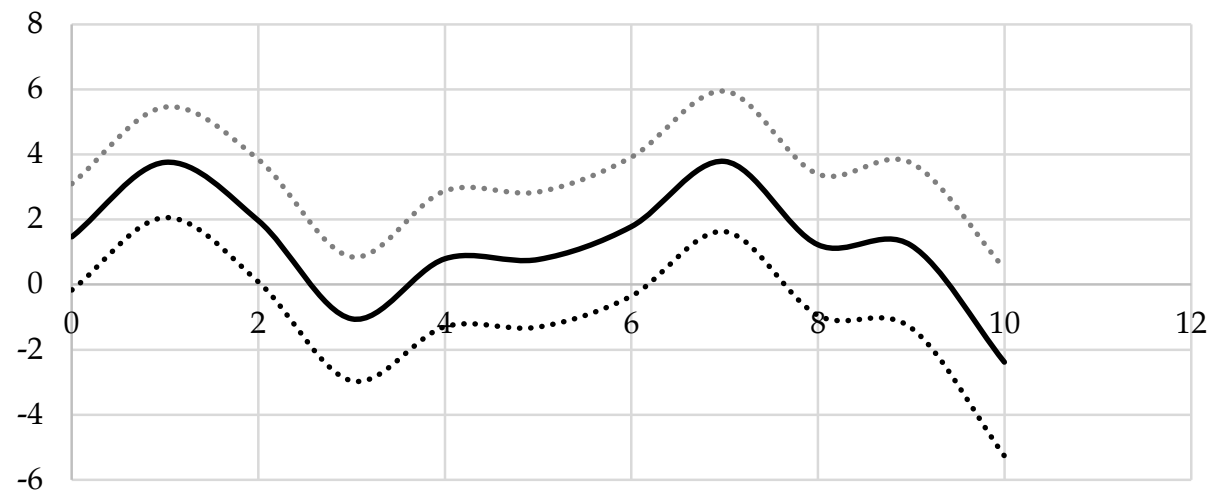

Note: The $x$-axis shows the number of time lags of firm entry or, in other words, the impact of firm entry one year later, two years later, etc. The y-axis shows the percentage change. Source: These figures are generated from the regression results shown above, based on data from the Punjab Directory of Industries. 


\section{Figure 7: Impact of large firm entry on district-level employment in} Punjab

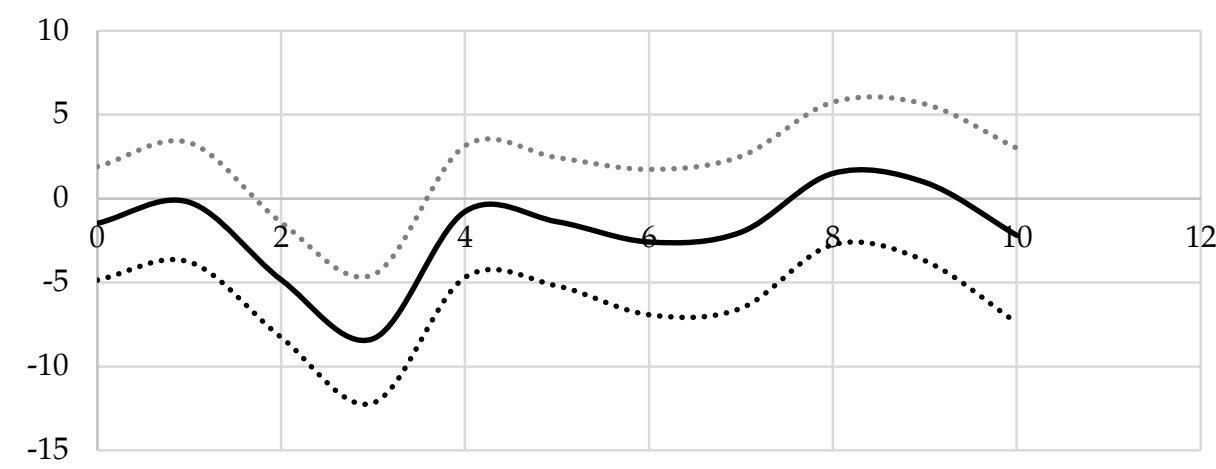

- Impact of firm entry on Change in Number of Hospitals

Note: The x-axis shows the number of time lags of firm entry or, in other words, the impact of firm entry one year later, two years later, etc. The y-axis shows the percentage change. Source: These figures are generated from the regression results shown above, based on data from the Punjab Directory of Industries.

The third set of results in Table 3 analyzes the impact of entrants that produce exportable goods on employment growth over different time periods. The results show that the entry of export good producers has a significant positive impact on employment growth; this impact is significant across multiple periods. Specifically, employment growth increases a year after the entry of export good-producing firms and this persists three, five, six, and seven years later. The entry of export good producers thus has a significant and persistent positive impact over time. 
Table 3: Impact of export good-producing firm entry on employment across districts in Punjab

\begin{tabular}{|c|c|c|c|}
\hline \multirow[b]{2}{*}{ Variable } & \multicolumn{3}{|c|}{ Employment growth } \\
\hline & $\begin{array}{l}2005-08 \\
\text { (1) }\end{array}$ & $\begin{array}{l}2007-10 \\
\text { (2) }\end{array}$ & $\begin{array}{c}2006-10 \\
\text { (3) }\end{array}$ \\
\hline Population density & $\begin{array}{c}0.0127 \\
(0.0672)\end{array}$ & $\begin{array}{l}-0.0347 \\
(0.0615)\end{array}$ & $\begin{array}{c}0.0280 \\
(0.0348)\end{array}$ \\
\hline Average firm size & $\begin{array}{c}-0.122^{*} \\
(0.0632)\end{array}$ & $\begin{array}{c}-0.443^{* *} \\
(0.198)\end{array}$ & $\begin{array}{l}-0.132^{* *} \\
(0.0629)\end{array}$ \\
\hline Firm birth $\mathrm{t}$ & $\begin{array}{c}-0.223 \\
(0.263)\end{array}$ & $\begin{array}{l}0.0692 \\
(0.315)\end{array}$ & $\begin{array}{l}0.0207 \\
(0.230)\end{array}$ \\
\hline Firm birth $\mathrm{t}-1$ & $\begin{array}{l}0.486^{*} \\
(0.291)\end{array}$ & $\begin{array}{c}0.418 \\
(0.268)\end{array}$ & $\begin{array}{l}0.431^{*} \\
(0.225)\end{array}$ \\
\hline Firm birth $\mathrm{t}-2$ & $\begin{array}{c}0.151 \\
(0.203)\end{array}$ & $\begin{array}{c}0.191 \\
(0.313)\end{array}$ & $\begin{array}{l}-0.268 \\
(0.198)\end{array}$ \\
\hline Firm birth $\mathrm{t}-3$ & $\begin{array}{l}0.433^{*} \\
(0.239)\end{array}$ & $\begin{array}{c}-0.454 \\
(0.289)\end{array}$ & $\begin{array}{l}0.0549 \\
(0.157)\end{array}$ \\
\hline Firm birth $\mathrm{t}-4$ & $\begin{array}{c}0.311 \\
(0.232)\end{array}$ & $\begin{array}{c}-0.244 \\
(0.186)\end{array}$ & $\begin{array}{c}-0.166 \\
(0.168)\end{array}$ \\
\hline Firm birth $\mathrm{t}-5$ & $\begin{array}{l}0.427^{* *} \\
(0.208)\end{array}$ & $\begin{array}{c}-0.519^{* *} \\
(0.245)\end{array}$ & $\begin{array}{l}-0.147 \\
(0.152)\end{array}$ \\
\hline Firm birth $\mathrm{t}-6$ & $\begin{array}{l}0.351^{* *} \\
(0.177)\end{array}$ & $\begin{array}{c}-0.324 \\
(0.224)\end{array}$ & $\begin{array}{l}-0.159 \\
(0.136)\end{array}$ \\
\hline Firm birth $\mathrm{t}-7$ & $\begin{array}{l}0.300^{*} \\
(0.171)\end{array}$ & $\begin{array}{l}-0.370^{*} \\
(0.195)\end{array}$ & $\begin{array}{l}-0.113 \\
(0.132)\end{array}$ \\
\hline Firm birth $\mathrm{t}-8$ & $\begin{array}{c}0.116 \\
(0.311)\end{array}$ & $\begin{array}{l}-0.331^{*} \\
(0.181)\end{array}$ & $\begin{array}{l}-0.0620 \\
(0.125)\end{array}$ \\
\hline Firm birth $\mathrm{t}-9$ & $\begin{array}{l}-0.115 \\
(0.308)\end{array}$ & $\begin{array}{l}-0.306^{*} \\
(0.165)\end{array}$ & $\begin{array}{l}-0.124 \\
(0.129)\end{array}$ \\
\hline Firm birth $\mathrm{t}-10$ & $\begin{array}{c}0.0775 \\
(0.0859)\end{array}$ & $\begin{array}{c}0.105 \\
(0.304)\end{array}$ & $\begin{array}{l}0.0670 \\
(0.220)\end{array}$ \\
\hline Constant & $\begin{array}{l}-13.57 \\
(41.52)\end{array}$ & $\begin{array}{c}67.85 \\
(46.19)\end{array}$ & $\begin{array}{l}-3.787 \\
(22.93)\end{array}$ \\
\hline R-squared & 0.113 & 0.195 & 0.085 \\
\hline District fixed effects & Yes & Yes & Yes \\
\hline
\end{tabular}

Note: Standard errors in parentheses.

${ }^{* * *} \mathrm{p}<0.01,{ }^{* *} \mathrm{p}<0.05,{ }^{*} \mathrm{p}<0.1$

Dependent variable

Employment growth $=$ (employment in region $i$ at time $t-$ employment in region $i$ at time $t-2$ )/employment in region $i$ at time $t-2$

Independent variables

Firm birth $=($ new firms producing export goods in region $i$ at time $\mathrm{t} /$ total new firms in Punjab at time $t$ )

Population density $=$ (population in region $i$ at time $t /$ area of a region $)$

Average firm size $=($ average size of firms in region $i$ at time $t)$

Source: Authors' calculations. 
Figure 8 shows that, as an exporting firm enters, the rate of change in employment increases by about 0.5 percent after a year; this impact persists over many years.

Figure 8: Impact of entry of exporting firms on district-level employment in Punjab, 2005-08

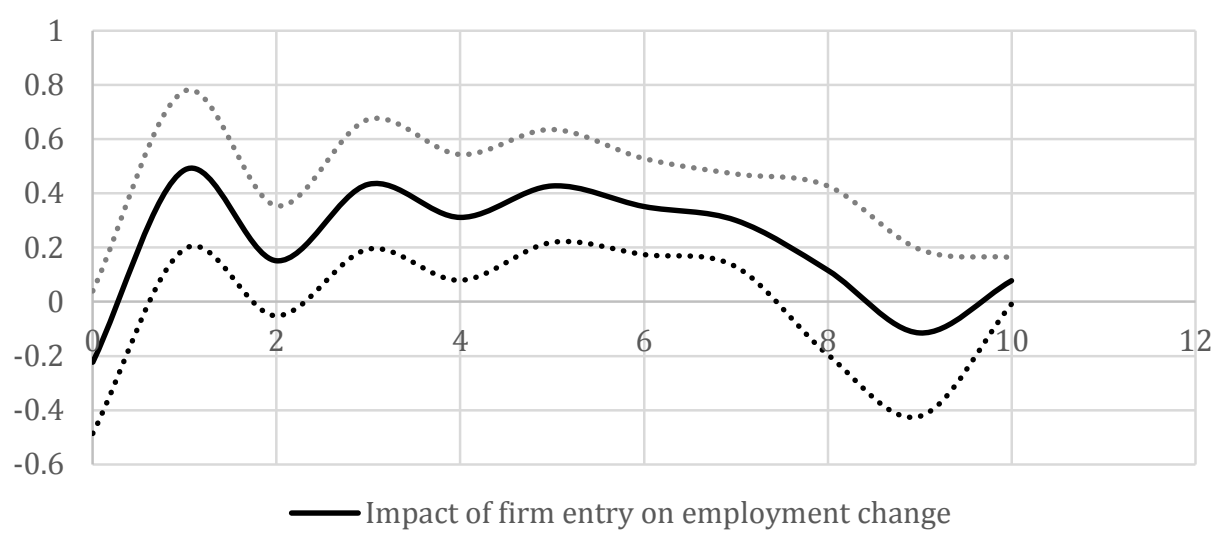

Note: The $x$-axis shows the number of time lags of firm entry or, in other words, the impact of firm entry one year later, two years later, etc. The y-axis shows the percentage change. Source: These figures are generated from the regression results shown above, based on data from the Punjab Directory of Industries.

Next, we look at how firm entry affects district-level school enrollment and the number of schools in a district over time. The results in Table 4 show that firm entry has a significant and positive impact on both variables. The impact of firm entry takes an average of about two years to materialize in either case and the impact on the number of schools persists over time. 
Table 4: Impact of firm entry on educational outcomes across districts in Punjab

\begin{tabular}{lcccc}
\hline \multirow{2}{*}{ Variable } & \multicolumn{2}{c}{ Number of primary schools } & \multicolumn{2}{c}{ Primary school enrollment } \\
\cline { 2 - 5 } & $\mathbf{( 1 )}$ & $\mathbf{( 2 )}$ & $\mathbf{( 3 )}$ & $\mathbf{( 4 )}$ \\
\hline Firm birth $\mathrm{t}$ & 0.427 & 0.205 & 7.939 & 7.124 \\
& $(0.753)$ & $(0.683)$ & $(14.19)$ & $(12.77)$ \\
Firm birth $\mathrm{t}-1$ & 1.013 & $1.334^{*}$ & -19.30 & -18.25 \\
& $(0.843)$ & $(0.735)$ & $(15.90)$ & $(13.74)$ \\
Firm birth $\mathrm{t}-2$ & $3.001^{* * *}$ & $3.190^{* * *}$ & $39.56^{*}$ & $39.10^{*}$ \\
& $(1.105)$ & $(1.077)$ & $(20.83)$ & $(20.14)$ \\
Firm birth $\mathrm{t}-3$ & -1.033 & -0.973 & -12.51 & -11.69 \\
& $(1.632)$ & $(1.587)$ & $(30.76)$ & $(29.66)$ \\
Firm birth $\mathrm{t}-4$ & $3.292^{* * *}$ & $3.381^{* * *}$ & 15.17 & 14.27 \\
& $(1.045)$ & $(0.980)$ & $(19.71)$ & $(18.32)$ \\
Firm birth $\mathrm{t}-5$ & $1.937^{* *}$ & $1.848^{* *}$ & -22.38 & -22.79 \\
& $(0.880)$ & $(0.861)$ & $(16.60)$ & $(16.09)$ \\
Firm birth $\mathrm{t}-6$ & $2.381^{* * *}$ & $2.536^{* * *}$ & -12.70 & -11.82 \\
& $(0.676)$ & $(0.592)$ & $(12.75)$ & $(11.07)$ \\
Firm birth $\mathrm{t}-7$ & 0.256 & 0.147 & 1.580 & 2.048 \\
& $(0.734)$ & $(0.670)$ & $(13.84)$ & $(12.52)$ \\
Firm birth $\mathrm{t}-8$ & 1.236 & 1.064 & -23.25 & -22.65 \\
& $(1.082)$ & $(0.996)$ & $(20.40)$ & $(18.62)$ \\
Firm birth $\mathrm{t}-9$ & -0.382 & & -2.309 & \\
Firm birth $\mathrm{t}-10$ & $(0.839)$ & & $(15.81)$ & \\
& 1.287 & & -0.328 & \\
Constant & $(1.573)$ & & $(29.66)$ & \\
& -209.6 & -89.78 & $158,703^{* * *}$ & $157,947^{* * *}$ \\
\hline R-squared & $(612.9)$ & $(448.8)$ & $(11,554)$ & $(8,390)$ \\
District fixed effects & 0.336 & 0.325 & 0.150 & 0.149 \\
\hline & Yes & Yes & Yes & Yes \\
\hline
\end{tabular}

Note: Standard errors in parentheses.

${ }^{* * *} \mathrm{p}<0.01,{ }^{* *} \mathrm{p}<0.05,{ }^{*} \mathrm{p}<0.1$

Dependent variables

Number of primary schools $=$ total number of primary schools in region $i$ at time $t /$ total number of schools in Punjab at time $t$

Primary school enrollment $=$ total number of students enrolled at primary level in region $i$ at time $t$ /total number of students enrolled in Punjab at time $t$

Independent variable

Firm birth $=($ new firms in region $i$ at time $t /$ total new firms in Punjab at time $t)$

Source: Authors' calculations.

Figures 9 and 10 show that the entry of a new firm increases enrollment rates and the number of schools after about a year. While this 
impact persists over time for the number of schools, the impact on primary enrollment rates becomes insignificant after a year.

\section{Figure 9: Impact of all firm entry on the number of primary schools at the district level in Punjab}

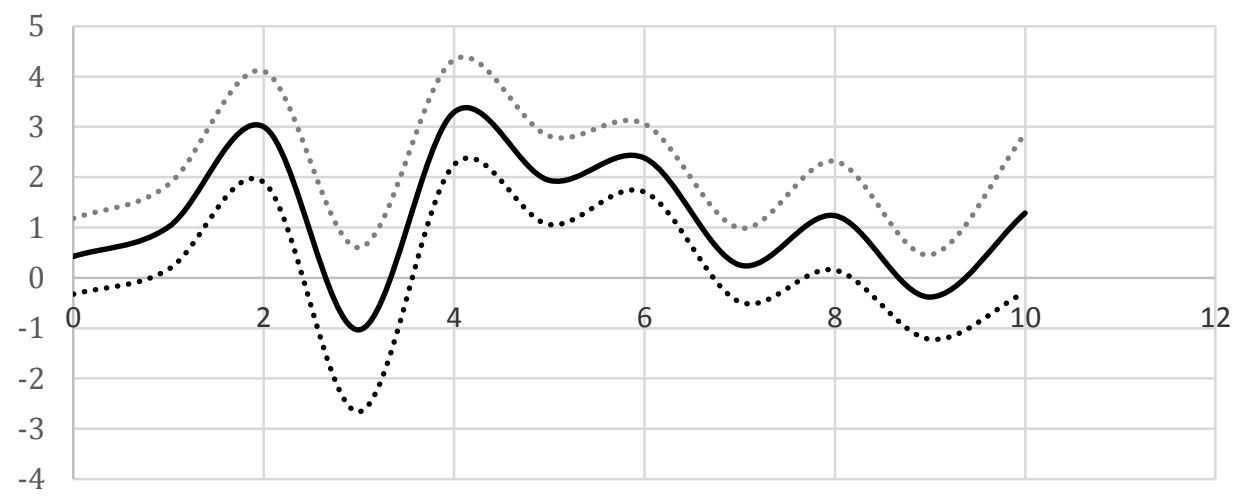

Note: The $x$-axis shows the number of time lags of firm entry or, in other words, the impact of firm entry one year later, two years later, etc. The y-axis shows the percentage change. Source: These figures are generated from the regression results shown above, based on data from the Punjab Directory of Industries.

\section{Figure 10: Impact of all firm entry on primary enrollment at the district} level in Punjab

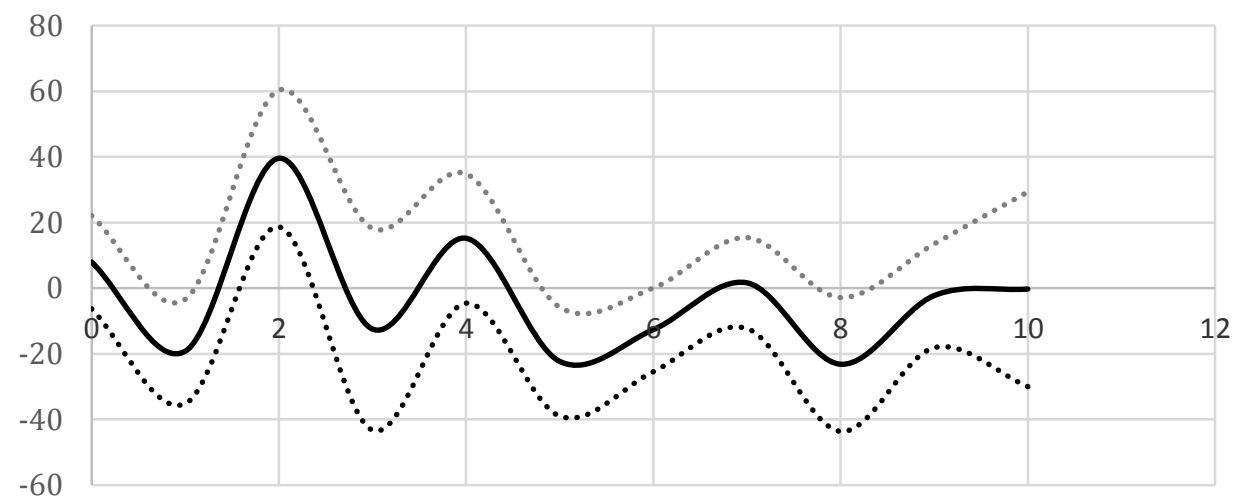

Note: The x-axis shows the number of time lags of firm entry or, in other words, the impact of firm entry one year later, two years later, etc. The y-axis shows the percentage change. Source: These figures are generated from the regression results shown above, based on data from the Punjab Directory of Industries.

Table 5 shows the impact of firm entry on the number of hospitals in a district over time. The results are similar to those for the educational outcomes discussed above. When new firms enter a district, there is an 
increase in the number of hospitals. This impact, while initially negative, becomes positive after a year and persists for up to three years.

Table 5: Impact of firm entry on number of hospitals across districts in Punjab

\begin{tabular}{lcc}
\hline & \multicolumn{2}{c}{ Number of hospitals } \\
\cline { 2 - 3 } Variable & $\mathbf{( 1 )}$ & $\mathbf{( 2 )}$ \\
\hline Firm birth $\mathrm{t}$ & $-2.321^{*}$ & $-2.362^{*}$ \\
& $(1.361)$ & $(1.405)$ \\
Firm birth $\mathrm{t}-1$ & $3.403^{*}$ & 2.610 \\
& $(1.716)$ & $(2.370)$ \\
Firm birth $\mathrm{t}-2$ & 2.021 & 3.226 \\
& $(1.554)$ & $(2.921)$ \\
Firm birth $\mathrm{t}-3$ & $3.639^{* *}$ & $4.790^{*}$ \\
& $(1.487)$ & $(2.732)$ \\
Firm birth $\mathrm{t}-4$ & & 2.182 \\
& & $(3.129)$ \\
Firm birth $\mathrm{t}-5$ & & -0.118 \\
& & $(4.714)$ \\
Constant & $8.520^{* * *}$ & $8.194^{* * *}$ \\
& $(0.466)$ & $(1.438)$ \\
\hline R-squared & 0.142 & 0.149 \\
District fixed effects & Yes & Yes \\
\hline
\end{tabular}

Note: Standard errors in parentheses.

${ }^{* * *} \mathrm{p}<0.01,{ }^{* *} \mathrm{p}<0.05,{ }^{*} \mathrm{p}<0.1$

Dependent variable

Number of hospitals $=$ total number of hospitals in region $i$ at time $t$ /total number of hospitals in Punjab at time $t$

Independent variable

Firm birth $=($ new firms in region $i$ at time $t /$ total new firms in Punjab at time $t)$

Source: Authors' calculations.

Tables 6 and 7 look at the impact of the entry of small, medium, and large firms on the number of schools and primary enrollment rates, and on the number of hospitals, respectively. Table 6 indicates that the entry of small and medium firms leads to a decline in the enrollment rate early on (after about three years). Subsequently, however, the enrollment rate rises, on average seven years after initial firm entry. The entry of large firms leads to the highest increase in primary enrollment, although it takes about four years for this impact to occur. Table 7 shows that the entry of large firms is associated with a marginal increase in the number of hospitals. The 
entry of small and medium firms is correlated with a marginal decrease in the number of hospitals.

Table 6: Impact of small, medium, and large firm entry on schooling outcomes across districts in Punjab

\begin{tabular}{|c|c|c|}
\hline \multirow[b]{2}{*}{ Variable } & Number of primary schools & Primary school enrollment \\
\hline & (1) & (2) \\
\hline \multirow[t]{2}{*}{ Small firm birth $\mathrm{t}$} & 0.805 & -21.01 \\
\hline & $(0.814)$ & $(13.76)$ \\
\hline \multirow[t]{2}{*}{ Small firm birth $\mathrm{t}-1$} & 0.225 & 2.088 \\
\hline & $(0.661)$ & (11.16) \\
\hline \multirow[t]{2}{*}{ Small firm birth $\mathrm{t}-2$} & $1.443^{* *}$ & -12.23 \\
\hline & $(0.643)$ & $(10.86)$ \\
\hline \multirow[t]{2}{*}{ Small firm birth $t-3$} & $2.138^{* * *}$ & $-36.23^{* * *}$ \\
\hline & $(0.713)$ & $(12.05)$ \\
\hline \multirow[t]{2}{*}{ Small firm birth $\mathrm{t}-4$} & -0.776 & 8.314 \\
\hline & $(0.693)$ & $(11.70)$ \\
\hline \multirow[t]{2}{*}{ Small firm birth $t-5$} & 0.245 & -8.681 \\
\hline & $(0.561)$ & $(9.475)$ \\
\hline \multirow[t]{2}{*}{ Small firm birth $t-6$} & -0.108 & 1.102 \\
\hline & $(0.613)$ & $(10.36)$ \\
\hline \multirow[t]{2}{*}{ Small firm birth $\mathrm{t}-7$} & -0.218 & 0.647 \\
\hline & $(0.571)$ & $(9.639)$ \\
\hline \multirow[t]{2}{*}{ Small firm birth $\mathrm{t}-8$} & -0.934 & $23.75^{* *}$ \\
\hline & $(0.602)$ & $(10.17)$ \\
\hline \multirow[t]{2}{*}{ Small firm birth $t-9$} & 0.127 & -10.31 \\
\hline & $(0.459)$ & $(7.753)$ \\
\hline \multirow[t]{2}{*}{ Small firm birth $\mathrm{t}-10$} & 0.194 & 7.047 \\
\hline & $(0.612)$ & $(10.35)$ \\
\hline \multirow[t]{2}{*}{ Medium firm birth $t$} & $-1.074^{* *}$ & 6.987 \\
\hline & $(0.499)$ & $(8.423)$ \\
\hline \multirow[t]{2}{*}{ Medium firm birth $\mathrm{t}-1$} & 0.263 & -11.70 \\
\hline & $(0.463)$ & $(7.821)$ \\
\hline \multirow[t]{2}{*}{ Medium firm birth $\mathrm{t}-2$} & 0.106 & -8.189 \\
\hline & $(0.498)$ & $(8.418)$ \\
\hline \multirow[t]{2}{*}{ Medium firm birth $t-3$} & -0.252 & $-18.40^{*}$ \\
\hline & $(0.609)$ & $(10.29)$ \\
\hline \multirow[t]{2}{*}{ Medium firm birth $t-4$} & $1.843^{* *}$ & -13.21 \\
\hline & $(0.701)$ & $(11.84)$ \\
\hline \multirow[t]{2}{*}{ Medium firm birth $t-5$} & -0.0256 & -7.214 \\
\hline & $(0.525)$ & $(8.873)$ \\
\hline Medium firm birth $t-6$ & -0.366 & 0.555 \\
\hline
\end{tabular}




\begin{tabular}{|c|c|c|}
\hline \multirow[b]{2}{*}{ Variable } & Number of primary schools & Primary school enrollment \\
\hline & (1) & $(2)$ \\
\hline \multirow{3}{*}{ Medium firm birth $\mathrm{t}-7$} & $(0.579)$ & $(9.784)$ \\
\hline & -0.0751 & $22.12^{*}$ \\
\hline & $(0.640)$ & $(10.81)$ \\
\hline \multirow[t]{2}{*}{ Medium firm birth $\mathrm{t}-8$} & -0.0472 & 0.378 \\
\hline & $(0.479)$ & $(8.100)$ \\
\hline \multirow[t]{2}{*}{ Medium firm birth $t-9$} & -0.825 & 2.452 \\
\hline & $(0.639)$ & $(10.79)$ \\
\hline \multirow[t]{2}{*}{ Medium firm birth $\mathrm{t}-10$} & -0.0623 & 7.396 \\
\hline & $(0.655)$ & $(11.07)$ \\
\hline \multirow[t]{2}{*}{ Large firm birth $t$} & -1.257 & 17.99 \\
\hline & $(1.126)$ & $(19.03)$ \\
\hline \multirow[t]{2}{*}{ Large firm birth $\mathrm{t}-1$} & 0.563 & -0.238 \\
\hline & $(1.033)$ & $(17.46)$ \\
\hline \multirow[t]{2}{*}{ Large firm birth $\mathrm{t}-2$} & 0.00279 & 1.695 \\
\hline & $(0.939)$ & $(15.86)$ \\
\hline \multirow[t]{2}{*}{ Large firm birth $t-3$} & 1.018 & -12.32 \\
\hline & $(0.985)$ & $(16.64)$ \\
\hline \multirow[t]{2}{*}{ Large firm birth $\mathrm{t}-4$} & $-3.040^{* *}$ & $52.74^{* *}$ \\
\hline & $(1.186)$ & $(20.04)$ \\
\hline \multirow[t]{2}{*}{ Large firm birth $t-5$} & -0.451 & 17.34 \\
\hline & $(0.845)$ & $(14.28)$ \\
\hline \multirow[t]{2}{*}{ Large firm birth $t-6$} & 0.277 & -9.625 \\
\hline & $(1.209)$ & $(20.42)$ \\
\hline \multirow[t]{2}{*}{ Large firm birth $\mathrm{t}-7$} & -0.991 & -12.66 \\
\hline & $(1.039)$ & $(17.56)$ \\
\hline \multirow[t]{2}{*}{ Large firm birth $\mathrm{t}-8$} & -0.780 & -10.69 \\
\hline & $(1.009)$ & $(17.05)$ \\
\hline \multirow[t]{2}{*}{ Large firm birth $t-9$} & 0.234 & -12.16 \\
\hline & $(1.426)$ & $(24.09)$ \\
\hline \multirow[t]{2}{*}{ Large firm birth $\mathrm{t}-10$} & 0.216 & -14.13 \\
\hline & $(0.945)$ & $(15.97)$ \\
\hline \multirow[t]{2}{*}{ Other firm birth $t$} & -2.224 & 14.66 \\
\hline & $(1.474)$ & $(24.90)$ \\
\hline \multirow[t]{2}{*}{ Other firm birth $\mathrm{t}-1$} & -3.390 & 23.74 \\
\hline & $(3.086)$ & $(52.13)$ \\
\hline \multirow[t]{2}{*}{ Other firm birth $\mathrm{t}-2$} & 0.184 & 0.233 \\
\hline & $(2.287)$ & $(38.64)$ \\
\hline \multirow[t]{2}{*}{ Other firm birth $\mathrm{t}-3$} & -3.414 & 27.66 \\
\hline & $(2.387)$ & $(40.33)$ \\
\hline \multirow[t]{2}{*}{ Other firm birth $\mathrm{t}-4$} & -2.766 & -184.2 \\
\hline & $(14.70)$ & $(248.4)$ \\
\hline
\end{tabular}




\begin{tabular}{lcc}
\hline & Number of primary schools & Primary school enrollment \\
\cline { 2 - 3 } Variable & $\mathbf{( 1 )}$ & $\mathbf{( 2 )}$ \\
\hline Other firm birth $\mathrm{t}-5$ & -0.927 & 34.63 \\
& $(2.539)$ & $(42.89)$ \\
Other firm birth $\mathrm{t}-6$ & 2.981 & 29.59 \\
& $(5.916)$ & $(99.95)$ \\
Other firm birth $\mathrm{t}-7$ & -3.904 & 49.36 \\
& $(4.075)$ & $(68.84)$ \\
Other firm birth $\mathrm{t}-8$ & -0.312 & 47.76 \\
& $(2.831)$ & $(47.82)$ \\
Other firm birth $\mathrm{t}-9$ & 2.269 & -18.35 \\
& $(2.086)$ & $(35.24)$ \\
Other firm birth $\mathrm{t}-10$ & -0.659 & 65.42 \\
& $(3.142)$ & $(53.09)$ \\
Constant & 1.597 & $166.7^{* * *}$ \\
& $(1.566)$ & $(26.45)$ \\
\hline R-squared & 0.693 & 0.684 \\
District fixed effects & Yes & Yes \\
\hline
\end{tabular}

Note: Standard errors in parentheses.

*** $\mathrm{p}<0.01,{ }^{* *} \mathrm{p}<0.05,{ }^{*} \mathrm{p}<0.1$

Dependent variables

Number of primary schools $=$ total number of primary schools in region $i$ at time $t /$ total number of schools in Punjab at time $t$

Primary school enrollment $=$ total number of students enrolled at primary level in region $i$ at time $t$ / total number of students enrolled in Punjab at time $t$

Independent variables

Small firm birth $=($ new firms with fewer than 10 employees in region $i$ at time $t$ / total new small firms in Punjab at time $t$ )

Medium firm birth $=$ (new firms with 10 or more employees and fewer than 50 in region $i$ at time $t$ /total new medium firms in Punjab at time $t$ )

Large firm birth $=$ (new firms with 50 or more employees in region $i$ at time $t /$ total new large firms in Punjab at time $t$ )

Other firm birth $=$ (new firms with no employment reported in region $i$ at time $t /$ total new firms whose employment is not reported in Punjab at time $t$ )

Source: Authors' calculations. 
Table 7: Impact of small, medium, and large firm entry on number of hospitals across districts in Punjab

\begin{tabular}{|c|c|}
\hline Variable & Number of hospitals \\
\hline \multirow[t]{2}{*}{ Small firm birth $t$} & -0.000335 \\
\hline & $(0.00104)$ \\
\hline \multirow[t]{2}{*}{ Small firm birth $\mathrm{t}-1$} & $-6.43 e-05$ \\
\hline & $(0.00120)$ \\
\hline \multirow[t]{2}{*}{ Small firm birth $\mathrm{t}-2$} & 0.000485 \\
\hline & $(0.00140)$ \\
\hline \multirow[t]{2}{*}{ Small firm birth $t-3$} & 0.00127 \\
\hline & $(0.00132)$ \\
\hline \multirow[t]{2}{*}{ Small firm birth $\mathrm{t}-4$} & 0.00207 \\
\hline & $(0.00157)$ \\
\hline \multirow[t]{2}{*}{ Small firm birth $t-5$} & 0.00220 \\
\hline & $(0.00135)$ \\
\hline \multirow[t]{2}{*}{ Small firm birth $t-6$} & 0.000892 \\
\hline & $(0.00117)$ \\
\hline \multirow[t]{2}{*}{ Small firm birth $\mathrm{t}-7$} & -0.000281 \\
\hline & $(0.00113)$ \\
\hline \multirow[t]{2}{*}{ Small firm birth $\mathrm{t}-8$} & 0.000860 \\
\hline & $(0.00117)$ \\
\hline \multirow[t]{2}{*}{ Small firm birth $t-9$} & $-0.00179^{* *}$ \\
\hline & $(0.000817)$ \\
\hline \multirow[t]{2}{*}{ Small firm birth $\mathrm{t}-10$} & $8.77 \mathrm{e}-05$ \\
\hline & $(0.000934)$ \\
\hline \multirow[t]{2}{*}{ Medium firm birth $t$} & 0.000719 \\
\hline & $(0.000800)$ \\
\hline \multirow[t]{2}{*}{ Medium firm birth $\mathrm{t}-1$} & 0.000538 \\
\hline & $(0.000732)$ \\
\hline \multirow[t]{2}{*}{ Medium firm birth $\mathrm{t}-2$} & 0.00246 \\
\hline & $(0.00144)$ \\
\hline \multirow[t]{2}{*}{ Medium firm birth $\mathrm{t}-3$} & $0.00371^{* *}$ \\
\hline & $(0.00154)$ \\
\hline \multirow[t]{2}{*}{ Medium firm birth $\mathrm{t}-4$} & $0.00345^{*}$ \\
\hline & $(0.00174)$ \\
\hline \multirow[t]{2}{*}{ Medium firm birth $t-5$} & $0.00311^{* *}$ \\
\hline & $(0.00140)$ \\
\hline \multirow[t]{2}{*}{ Medium firm birth $\mathrm{t}-6$} & $0.00423^{* *}$ \\
\hline & $(0.00157)$ \\
\hline
\end{tabular}




\begin{tabular}{|c|c|}
\hline Variable & Number of hospitals \\
\hline \multirow[t]{2}{*}{ Medium firm birth $\mathrm{t}-7$} & $0.00267^{* *}$ \\
\hline & $(0.00129)$ \\
\hline \multirow[t]{2}{*}{ Medium firm birth $\mathrm{t}-8$} & 0.00167 \\
\hline & $(0.00103)$ \\
\hline \multirow[t]{2}{*}{ Medium firm birth $t-9$} & -0.000517 \\
\hline & $(0.000840)$ \\
\hline \multirow[t]{2}{*}{ Medium firm birth $\mathrm{t}-10$} & $5.49 \mathrm{e}-05$ \\
\hline & $(0.000900)$ \\
\hline \multirow[t]{2}{*}{ Large firm birth $t$} & 0.00203 \\
\hline & $(0.00264)$ \\
\hline \multirow[t]{2}{*}{ Large firm birth $\mathrm{t}-1$} & 0.00162 \\
\hline & $(0.00274)$ \\
\hline \multirow[t]{2}{*}{ Large firm birth $\mathrm{t}-2$} & 0.00216 \\
\hline & $(0.00255)$ \\
\hline \multirow[t]{2}{*}{ Large firm birth $\mathrm{t}-3$} & 0.000542 \\
\hline & $(0.00269)$ \\
\hline \multirow[t]{2}{*}{ Large firm birth $\mathrm{t}-4$} & -0.000984 \\
\hline & $(0.00240)$ \\
\hline \multirow[t]{2}{*}{ Large firm birth $\mathrm{t}-5$} & -0.00324 \\
\hline & $(0.00215)$ \\
\hline \multirow[t]{2}{*}{ Large firm birth $\mathrm{t}-6$} & -0.00269 \\
\hline & $(0.00227)$ \\
\hline \multirow[t]{2}{*}{ Large firm birth $\mathrm{t}-7$} & -0.000609 \\
\hline & $(0.00256)$ \\
\hline \multirow[t]{2}{*}{ Large firm birth $\mathrm{t}-8$} & -0.00375 \\
\hline & $(0.00250)$ \\
\hline \multirow[t]{2}{*}{ Large firm birth $\mathrm{t}-9$} & $-0.00511^{* *}$ \\
\hline & $(0.00242)$ \\
\hline \multirow[t]{2}{*}{ Large firm birth $\mathrm{t}-10$} & $-0.00457^{*}$ \\
\hline & $(0.00267)$ \\
\hline \multirow[t]{2}{*}{ Other firm birth $t$} & 0.00293 \\
\hline & $(0.00239)$ \\
\hline \multirow[t]{2}{*}{ Other firm birth $\mathrm{t}-1$} & 0.0138 \\
\hline & $(0.0114)$ \\
\hline \multirow[t]{2}{*}{ Other firm birth $\mathrm{t}-2$} & 0.00539 \\
\hline & $(0.0115)$ \\
\hline \multirow[t]{2}{*}{ Other firm birth $\mathrm{t}-3$} & -0.00117 \\
\hline & $(0.0120)$ \\
\hline
\end{tabular}




\begin{tabular}{lc}
\hline Variable & Number of hospitals \\
\hline Other firm birth $\mathrm{t}-4$ & -0.00309 \\
& $(0.0126)$ \\
Other firm birth $\mathrm{t}-5$ & -0.00744 \\
& $(0.0132)$ \\
Other firm birth $\mathrm{t}-6$ & -0.00602 \\
& $(0.00594)$ \\
Other firm birth $\mathrm{t}-7$ & 0.00904 \\
& $(0.00635)$ \\
Other firm birth $\mathrm{t}-8$ & 0.00872 \\
& $(0.00525)$ \\
Other firm birth $\mathrm{t}-9$ & $0.00906^{* * *}$ \\
& $(0.00315)$ \\
Other firm birth $\mathrm{t}-10$ & $0.00852^{* *}$ \\
& $(0.00404)$ \\
Constant & 0.00118 \\
& $(0.00428)$ \\
\hline R-squared & 0.882 \\
District fixed effects & Yes \\
\hline
\end{tabular}

Note: Standard errors in parentheses.

${ }^{* * *} \mathrm{p}<0.01,{ }^{* *} \mathrm{p}<0.05,{ }^{*} \mathrm{p}<0.1$

Dependent variable

Number of hospitals $=$ total number of hospitals in region $i$ at time $t /$ total number of hospitals in Punjab at time $t$

Independent variables

Small firm birth $=$ (new firms with fewer than 10 employees in region $i$ at time $t /$ total new small firms in Punjab at time $t)$

Medium firm birth $=$ (new firms with 10 or more employees and fewer than 50 in region $i$ at time $t /$ total new medium firms in Punjab at time $t)$

Large firm birth $=$ (new firms with 50 or more employees in region $i$ at time $t /$ total new large firms in Punjab at time $t$ )

Other firm birth $=($ new firms with no employment reported in region $i$ at time $t /$ total new firms whose employment is not reported in Punjab at time $t$ )

Source: Authors' calculations.

The results for the impact of firms of various sizes on schooling outcomes are shown in Figures 11-16. We observe that the entry of small firms does not significantly increase primary enrollment rates, while the entry of medium firms initially decreases district-level primary enrollment rates for almost five years, after which a positive impact on primary enrollment arises. The largest increase in primary enrollment rates is associated with the entry of large firms in a district, but this impact is only observed about four years after firm entry and is short-lived. 
Figure 11: Impact of small firm entry on primary school enrollment in Punjab

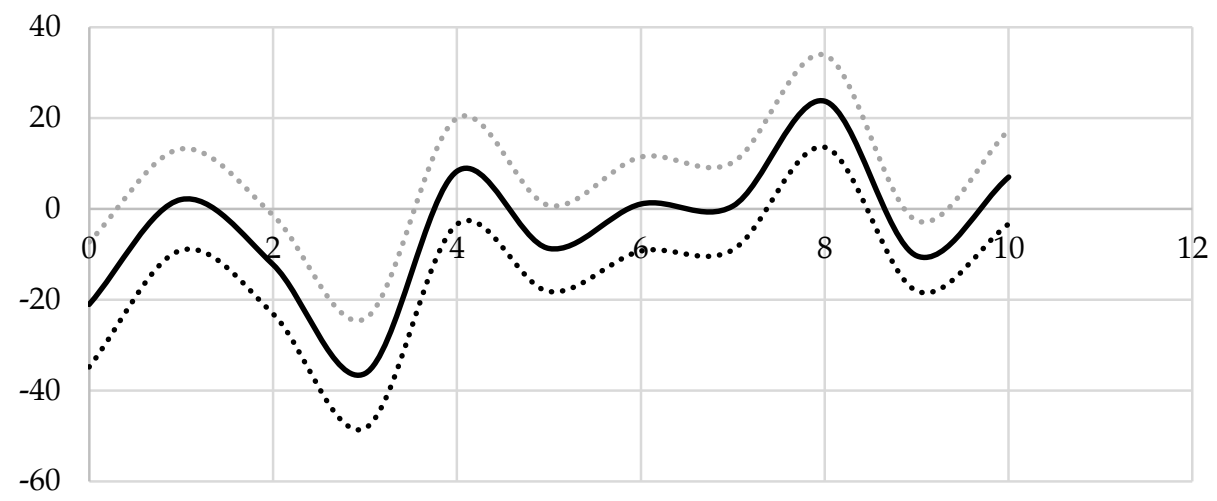

Note: The x-axis shows the number of time lags of firm entry or, in other words, the impact of firm entry one year later, two years later, etc. The y-axis shows the percentage change. Source: These figures are generated from the regression results shown above, based on data from the Punjab Directory of Industries.

\section{Figure 12: Impact of medium firm entry on primary school enrollment in Punjab}

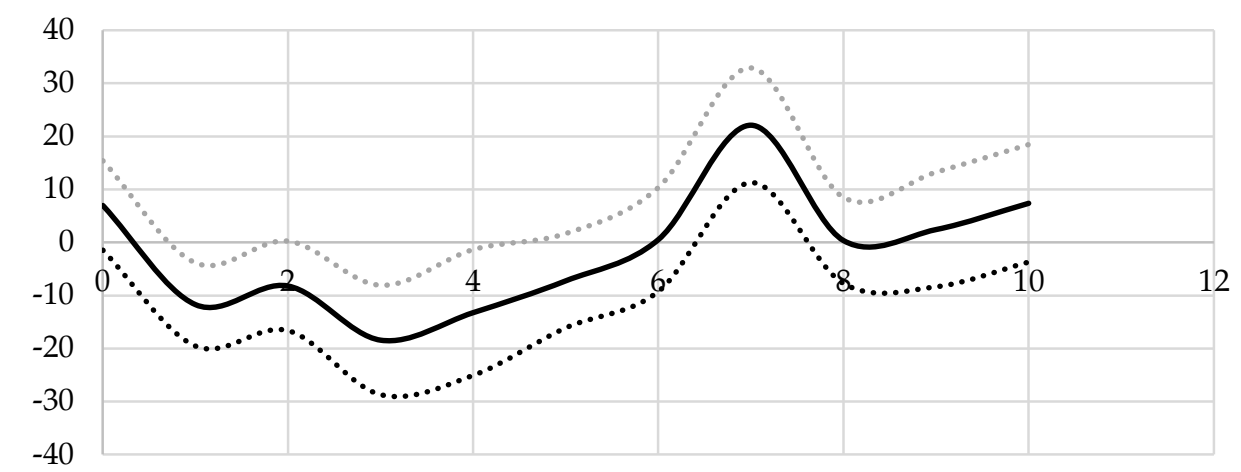

Note: The $x$-axis shows the number of time lags of firm entry or, in other words, the impact of firm entry one year later, two years later, etc. The y-axis shows the percentage change. Source: These figures are generated from the regression results shown above, based on data from the Punjab Directory of Industries. 
Figure 13: Impact of large firm entry on primary school enrollment in Punjab

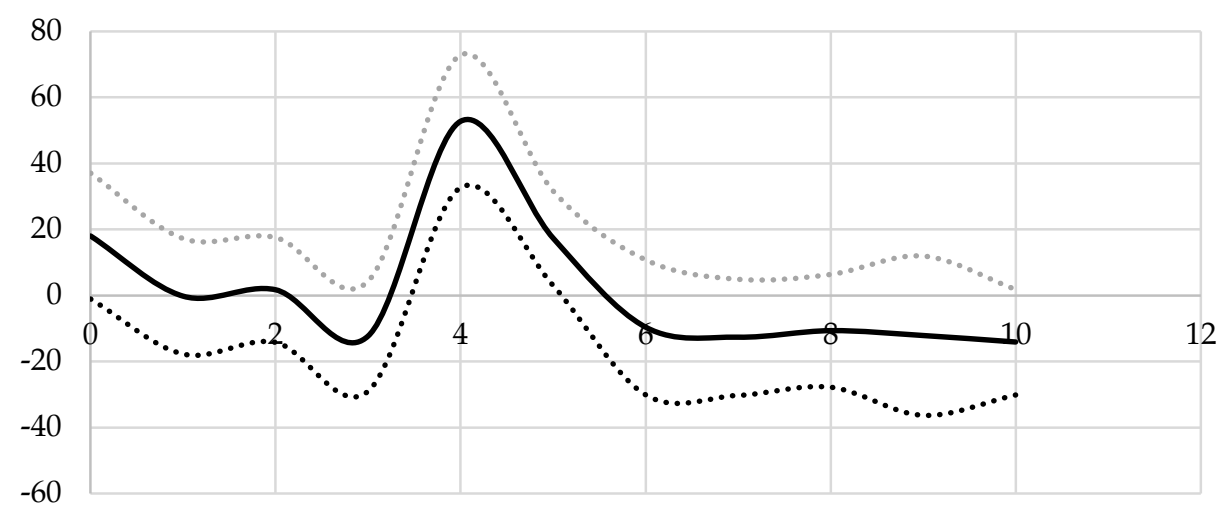

Note: The x-axis shows the number of time lags of firm entry or, in other words, the impact of firm entry one year later, two years later, etc. The y-axis shows the percentage change. Source: These figures are generated from the regression results shown above, based on data from the Punjab Directory of Industries.

\section{Figure 14: Impact of small firm entry on the number of primary schools in Punjab}

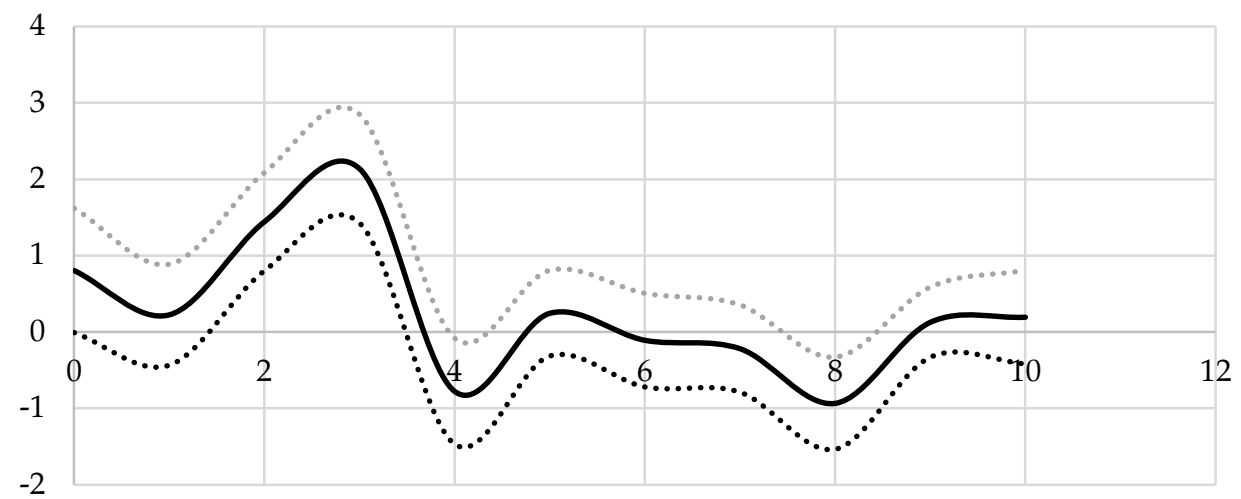

Note: The x-axis shows the number of time lags of firm entry or, in other words, the impact of firm entry one year later, two years later, etc. The y-axis shows the percentage change.

Source: These figures are generated from the regression results shown above, based on data from the Punjab Directory of Industries. 


\section{Figure 15: Impact of medium firm entry on the number of primary schools in Punjab}

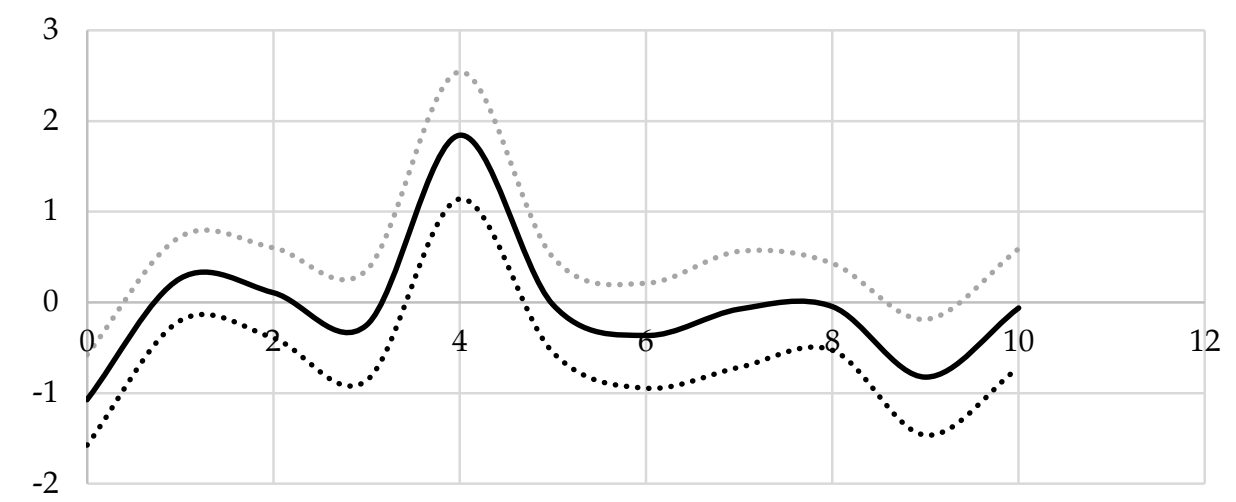

Note: The $x$-axis shows the number of time lags of firm entry or, in other words, the impact of firm entry one year later, two years later, etc. The y-axis shows the percentage change.

Source: These figures are generated from the regression results shown above, based on data from the Punjab Directory of Industries.

\section{Figure 16: Impact of large firm entry on the number of primary schools in Punjab}

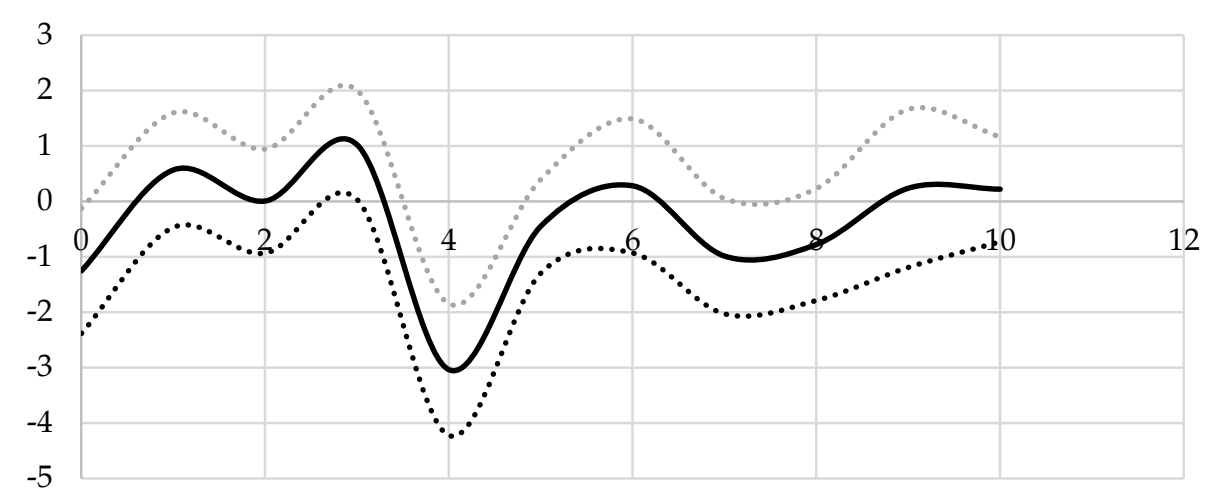

Note: The $x$-axis shows the number of time lags of firm entry or, in other words, the impact of firm entry one year later, two years later, etc. The y-axis shows the percentage change. Source: These figures are generated from the regression results shown above, based on data from the Punjab Directory of Industries.

Figures 17-19 illustrate the results for the impact of small, medium, and large firm entry on the number of hospitals in Punjab. 


\section{Figure 17: Impact of small firm entry on the number of hospitals in Punjab}

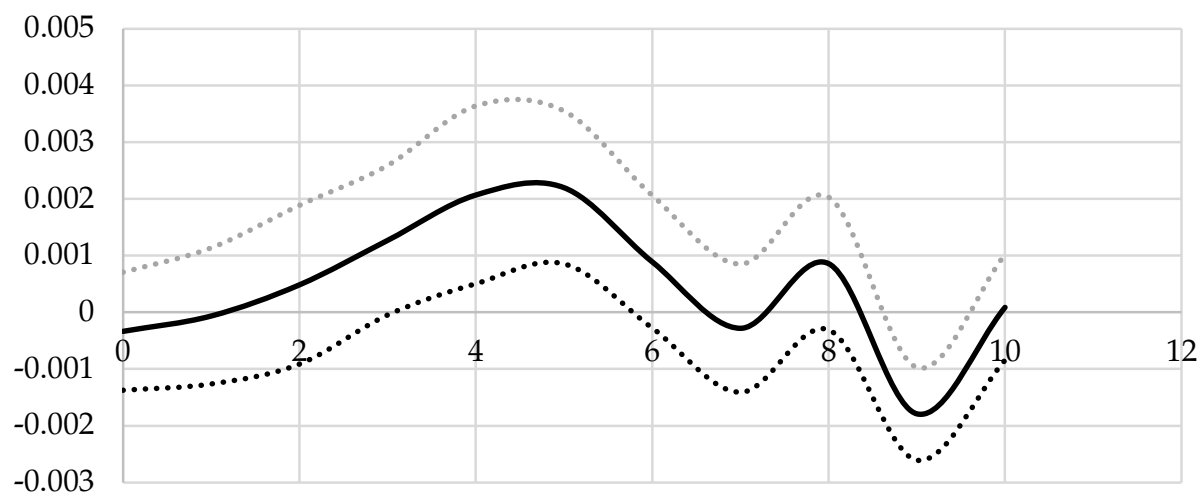

Note: The $x$-axis shows the number of time lags of firm entry or, in other words, the impact of firm entry one year later, two years later, etc. The y-axis shows the percentage change. Source: These figures are generated from the regression results shown above, based on data from the Punjab Directory of Industries.

\section{Figure 18: Impact of medium firm entry on the number of hospitals in Punjab}

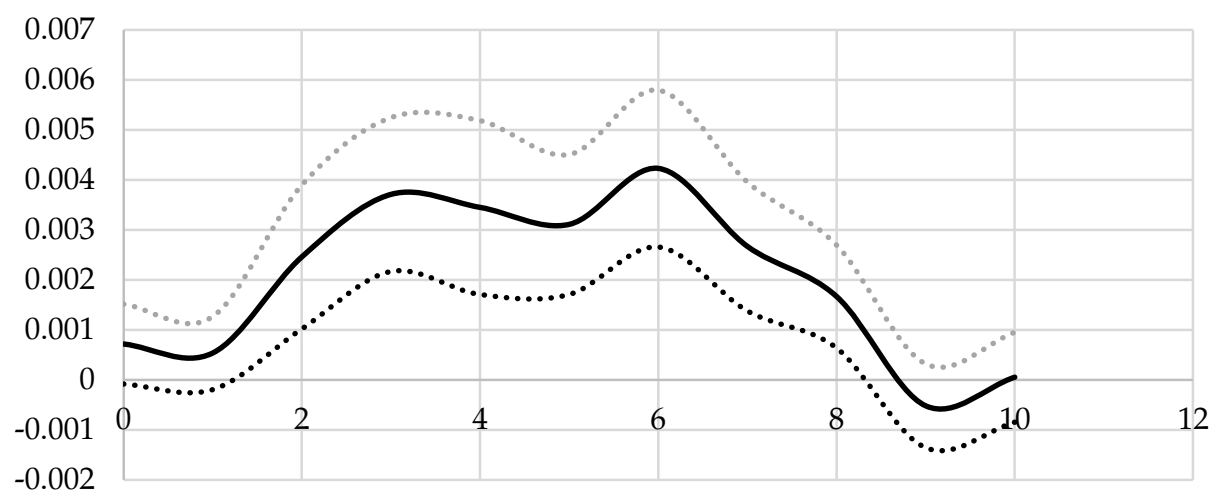

Note: The x-axis shows the number of time lags of firm entry or, in other words, the impact of firm entry one year later, two years later, etc. The y-axis shows the percentage change. Source: These figures are generated from the regression results shown above, based on data from the Punjab Directory of Industries. 
Figure 19: Impact of large firm entry on the number of hospitals in Punjab

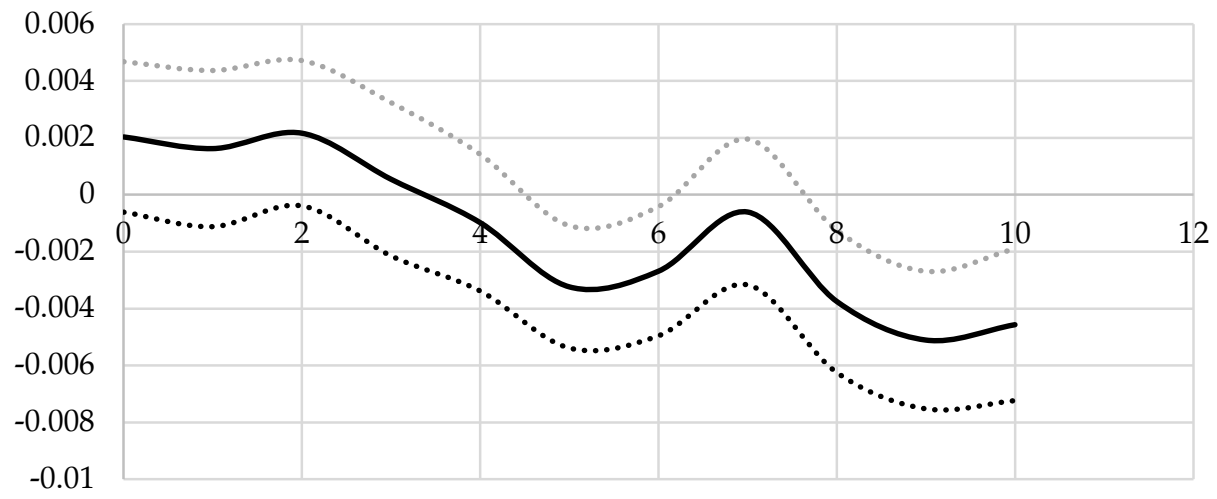

Note: The $x$-axis shows the number of time lags of firm entry or, in other words, the impact of firm entry one year later, two years later, etc. The y-axis shows the percentage change. Source: These figures are generated from the regression results shown above, based on data from the Punjab Directory of Industries.

Table 8 shows the impact of the entry of export producing firms on schooling outcomes across districts in Punjab. There is a significant increase in the number of schools and primary enrollment after the entry of an export-producing firm, but this is at least two years after entry in the case of the number of hospitals and at least six years in the case of districtlevel primary school enrollment. 
Table 8: Impact of export good-producing firm entry on schooling outcomes across districts in Punjab

\begin{tabular}{|c|c|c|}
\hline \multirow[b]{2}{*}{ Variable } & Number of primary schools & Primary school enrollment \\
\hline & $\begin{array}{c}(1) \\
\end{array}$ & (2) \\
\hline \multirow[t]{2}{*}{ Firm birth $\mathrm{t}$} & 0.104 & 2.690 \\
\hline & $(0.633)$ & $(11.91)$ \\
\hline \multirow[t]{2}{*}{ Firm birth $\mathrm{t}-1$} & 0.283 & -8.279 \\
\hline & $(0.786)$ & $(14.78)$ \\
\hline \multirow[t]{2}{*}{ Firm birth $\mathrm{t}-2$} & $2.037^{*}$ & -22.53 \\
\hline & $(1.020)$ & $(19.19)$ \\
\hline \multirow[t]{2}{*}{ Firm birth $\mathrm{t}-3$} & -0.199 & -9.052 \\
\hline & $(0.947)$ & $(17.82)$ \\
\hline \multirow[t]{2}{*}{ Firm birth $\mathrm{t}-4$} & $2.082^{* * *}$ & 3.674 \\
\hline & $(0.758)$ & $(14.27)$ \\
\hline \multirow[t]{2}{*}{ Firm birth $\mathrm{t}-5$} & 0.809 & 2.440 \\
\hline & $(1.646)$ & $(30.96)$ \\
\hline \multirow[t]{2}{*}{ Firm birth $\mathrm{t}-6$} & $2.992^{* *}$ & $43.59^{*}$ \\
\hline & $(1.353)$ & $(25.45)$ \\
\hline \multirow[t]{2}{*}{ Firm birth $\mathrm{t}-7$} & 0.0162 & -7.584 \\
\hline & $(0.983)$ & $(18.49)$ \\
\hline \multirow[t]{2}{*}{ Firm birth $\mathrm{t}-8$} & 0.0356 & -10.71 \\
\hline & $(0.961)$ & $(18.08)$ \\
\hline \multirow[t]{2}{*}{ Firm birth $\mathrm{t}-9$} & -0.217 & -19.62 \\
\hline & $(1.024)$ & $(19.27)$ \\
\hline \multirow[t]{2}{*}{ Firm birth $\mathrm{t}-10$} & -0.876 & 10.43 \\
\hline & $(1.284)$ & $(24.16)$ \\
\hline \multirow[t]{2}{*}{ Constant } & 0.625 & $154.1^{* * *}$ \\
\hline & $(0.755)$ & $(14.21)$ \\
\hline R-squared & 0.384 & 0.214 \\
\hline District fixed effects & Yes & Yes \\
\hline
\end{tabular}

Note: Standard errors in parentheses.

${ }^{* * *} \mathrm{p}<0.01,{ }^{* *} \mathrm{p}<0.05,{ }^{*} \mathrm{p}<0.1$

Dependent variables

Number of primary schools $=$ total number of primary schools in region $i$ at time $t /$ total number of schools in Punjab at time $t$

Primary school enrollment $=$ total number of students enrolled at primary level in region $i$ at time $t$ / total number of students enrolled in Punjab at time $t$

Independent variable

Firm birth $=($ new firms producing export goods in region $i$ at time $t /$ total new firms in Punjab at time $t$

Source: Authors' calculations. 
This is illustrated in Figures 20 and 21, which show how primary enrollment is positively affected about six years after the entry of an export-producing firm. The impact on the number of primary schools in a district increases after a few years and then fluctuates.

\section{Figure 20: Impact of entry of exporting firms on the number of primary schools in Punjab}

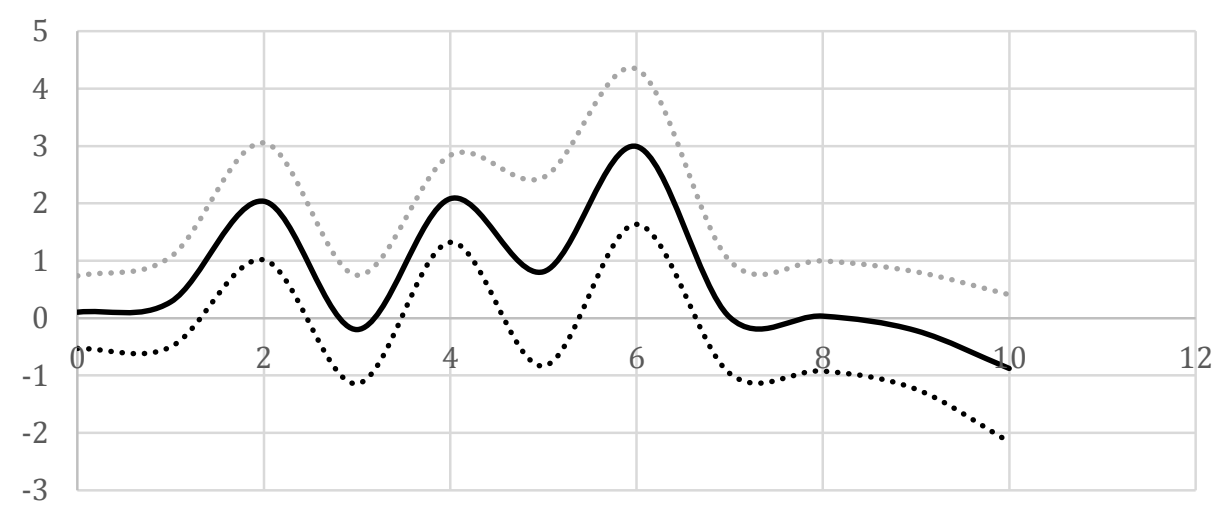

Note: The $x$-axis shows the number of time lags of firm entry or, in other words, the impact of firm entry one year later, two years later, etc. The y-axis shows the percentage change. Source: These figures are generated from the regression results shown above, based on data from the Punjab Directory of Industries.

Figure 21: Impact of entry of exporting firms on primary enrollment in Punjab

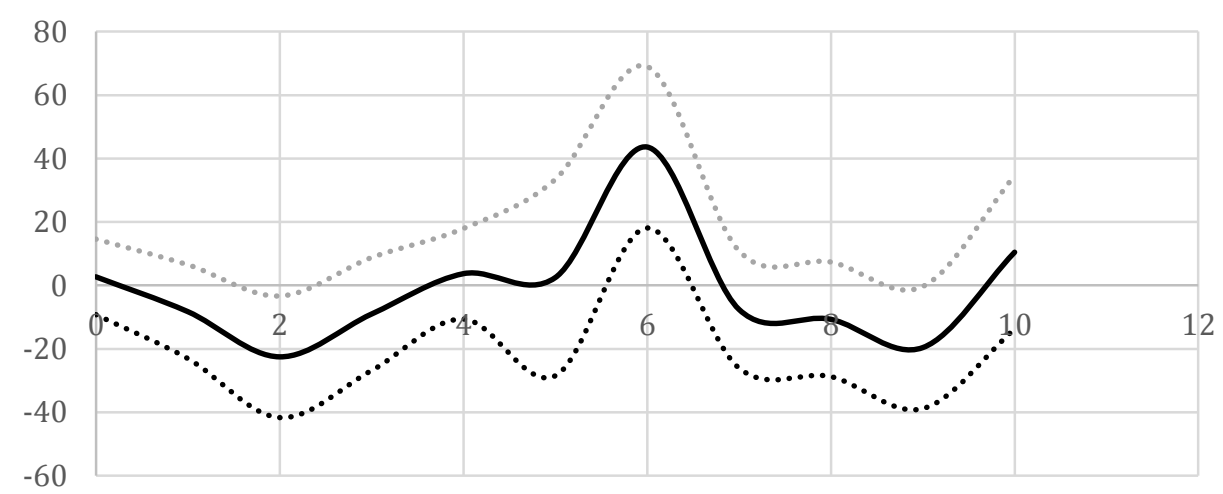

Note: The x-axis shows the number of time lags of firm entry or, in other words, the impact of firm entry one year later, two years later, etc. The $y$-axis shows the percentage change. Source: These figures are generated from the regression results shown above, based on data from the Punjab Directory of Industries. 


\section{Discussion}

Our analysis looks at the economic implications of new firm entry across the districts of Punjab. We start by looking at how new firm entry affects employment growth, primary enrollment rates, the number of schools, and the number of hospitals. We then extend the basic model to isolate the impact of firms of various sizes entering the market. Finally, we look at how export-oriented firm entry can have a different impact on the variables above compared to firms producing for the domestic market.

The results generally prove that the impact of new firm entry on employment growth fluctuates over time. When new firms enter the market, they initially cause an increase in employment - the direct employment effect - but this is followed by a decrease in district-level employment due to the displacement of workers as some firms are driven out of business. Eventually, employment goes up as the remaining firms perform better and expand.

The entry of small firms tends to lead to short-term increases in employment; this impact on employment is slightly longer-lived in the case of entry by medium firms. On average, there is a significant decrease in the growth rate of employment after a large firm enters the market and this impact is greater than that of a small firm. While the entry of export-oriented firms has a significant impact on employment that is sustained over time, this effect is substantially smaller than in the case of other types of firms.

In terms of socioeconomic outcomes - primary enrollment rates and the number of schools in a district over time - we find that overall firm entry tends to initially decrease primary enrollment rates in Punjab; this is followed by a pattern of increased and decreased enrollment rates over time. The entry of small firms has little impact on primary enrollment initially, but after a few years, it leads to a fall in primary enrollment, followed by gradual increases. The entry of medium firms leads to a significant decline in primary enrollment rates over time: this lasts almost seven years before becoming positive. The largest positive impact on primary enrollment is correlated with the entry of large firms, but it takes almost four years for this impact to materialize.

The entry of an export good producer also has a large, positive impact on primary school enrollment after about six years. It is tempting to believe that the results are similar because the largest firms and export good-producing firms are the same, but this is not necessarily the case: 
we do not get the same results for large firms and export producers when we look at the impact on employment. Finally, we find that new firm entry has a significant, if marginal, impact on the number of hospitals and primary schools.

Overall, our results imply that firm entry has a significant impact on socioeconomic outcomes, which differs across the economic variables we look at and also across the types of firms that enter the market. What is important to note is that some of these impacts are immediate while some take years to occur. In the context of formulating industrial policies, policymakers must recognize that different types of firms have different kinds of impacts, so that a one-size-fits-all approach to industrial development is unlikely to succeed. 


\section{References}

Acs, Z. J., \& Armington, C. (2003). Endogenous growth and entrepreneurship activity in cities (Working Paper No. 03-02). Washington, DC: Center for Economic Studies.

Acs, Z. J., \& Mueller, P. (2008). Employment effects of business dynamics: Mice, gazelles, and elephants. Small Business Economics, 30, 85-100.

Ashcroft, B., \& Love, J. H. (1996). Firm births and employment change in the British counties: 1981-1989. Papers in Regional Studies, 25, 483-500.

Baptista, R., \& Preto, M. T. (2011). New firm formation and employment growth: Regional and business dynamics. Small Business Economics, 36(4), 419-442.

Bosma, N., \& Nieuwenhuijsen, H. (2002). Turbulence and productivity; an analysis of 40 Dutch regions in the period 1988-1996. SCALES Paper N, 200205.

Brixy, U. (1999). Die Rolle von Betriebsgründungen für die Arbeitsplatzdynamik (The role of new businesses in employment dynamics) (Beiträge zur Arbeitsmarkt- und Berufsforschung, Bd. 230). Nuremberg: Bundesanstalt für Arbeit.

Callejón, M., \& Segarra, A. (2000). Business dynamics and efficiency in industries and regions. Small Business Economics, 13, 253-271.

Caves, R. E. (1998). Industrial organization and new findings on the turnover and mobility of firms. Journal of Economic Literature, 36, 1947-1982.

Chaudhry, T., \& Haseeb, M. (2014). Exporters in Pakistan and firms who do not export: What's the big difference? [Special edition]. Lahore Journal of Economics, 19, 207-246.

Fritsch, M., \& Mueller, P. (2004). Effects of new business formation on regional development over time. Regional Studies, 38(8), 961-975.

Fritsch, M., \& Mueller, P. (2008). The effect of new business formation on regional development over time: The case of Germany. Small Business Economics, 30, 15-29. 
Foelster, S. (2000). Do entrepreneurs create jobs? Small Business Economics, $14,137-148$.

Geroski, P. (1995). What do we know about entry? International Journal of Industrial Organization, 13, 421-440.

Mueller, P., van Stel, A., \& Storey, D. J. (2008). The effects of new firm formation on regional development over time: The case of Great Britain. Small Business Economics, 30, 59-71.

Reynolds, P. D. (1994). Autonomous firm dynamics and economic growth in the United States, 1986-90. Regional Studies, 27, 429-442.

Reynolds, P. D. (1999). Creative destruction: Source or symptom of economic growth? In Z. J. Acs, B. Carlsson, \& C. Karlsson (Eds.), Entrepreneurship, small and medium-sized enterprises and the macroeconomy (pp. 97-136). Cambridge: Cambridge University Press.

van Stel A., \& Suddle, K. (2008). The impact of new firm formation on regional development in the Netherlands. Small Business Economics, 30, 31-47. 